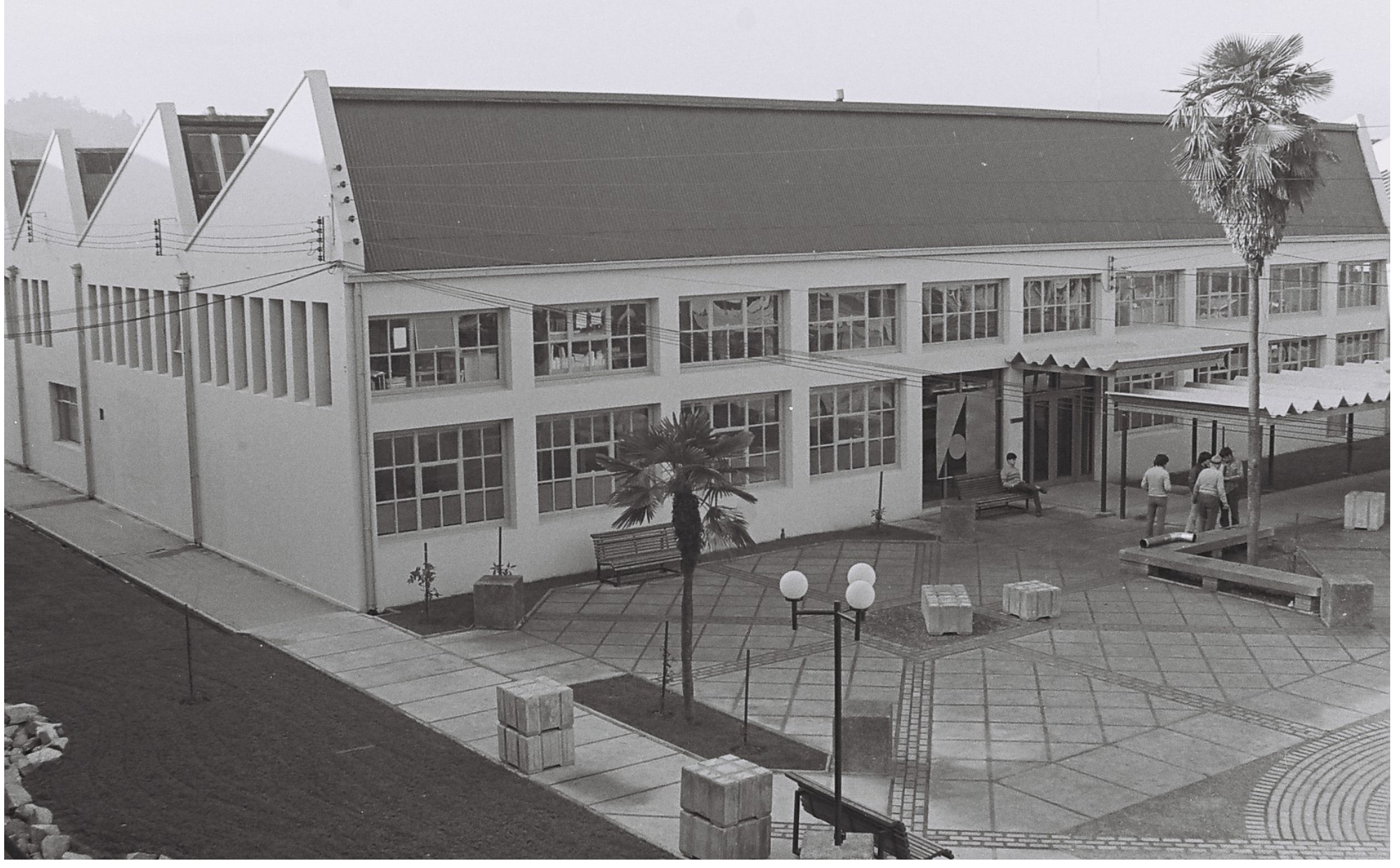

Figura 1. Edificio de la Escuela de Arquitectura, Universidad del Bío-Bío. Fuente: Archivo.

\title{
50 años formando profesionales de excelencia: los planes de estudios en la Escuela de Arquitectura, Universidad del Bio-Bio (1969-2014)
}

50 anos formando profissionais de excelência: os planos de estudo da Escola de Arquitetura, Universidade del Bío-Bío (1969-2014)

50 years training high-quality professionals: The plans of study at the School of Architecture, University of the Bio-Bio (1969-2014)

Flavio Valassina-Simonetta

Docente del Departamento de Diseño y Teoría de la Arquitectura, Universidad del Bío-Bío, Concepción, Chile fvalassi@ubiobio.cl | https://orcid.org/0000-0002-4716-8025 


\section{Resumen}

El artículo presenta la trayectoria de la carrera de Arquitectura de la UBB, desde su creación a la actualidad, a través de los ocho planes de estudios que marcaron la formación de las distintas cohortes profesionales que egresaron de esta escuela. La metología exploró la documentación existente en archivos desde su ideario fundacional a la fecha, también revisó los acuerdos que definieron el perfil de egreso y que identifican al arquitecto UBB, la construcción de los planes de estudios, de las mallas curriculares, y analizó la trayectoria de algunos de los profesores que materializaron en las aulas esos procesos de enseñanza en correspondencia con los acontecimientos nacionales y locales de esa época. Justamente, cuando esta Escuela cumple 50 años formando profesionales arquitectos de gran prestigio nacional, este recorrido por sus modelos pedagógicos reafirma el merecido tercer lugar que le fuera concedido entre las carreras de arquitectura en el país y revalida la acreditación internacional otorgada por el Royal Institute of British Architects (RIBA) desde el año 1991.

\section{Resumo}

0 artigo apresenta a trajetória do curso de Arquitetura da UBB desde sua criação até a atualidade através dos oitos planos de estudo que marcaram a formação de distintos grupos professionais egressos desta escola. A metodologia explorou a documentação existente em arquivos desde sua ideologia fundadora à atualidade, também revisou os acordos que definiram o perfil de egresso e que identificam o arquiteto UBB, a construção dos planos de estudo, das grades curriculares, e analizou a trajetória de alguns dos professores que materializaram em classe esses processos de ensino em correspondência com os acontecimentos nacionais e locais da época. Justamente, quando esta Escola completa 50 anos de trajetória formando profissionais arquitetos de grande perstigio nacional, esta incursão por seus modelos pedagógicos reafirma o merecido terceiro lugar que the foi concedido entre os cursos de arquitetura do país e revalida a acreditação internacional outorgada pela Royal Institute of British Architects (RIBA) desde 1991.

\section{Abstract}

This article presents the evolution of the Architecture degree program at the University of the Bio-Bio (UBB) from its creation to the present, through the eight plans of study that have marked the training of the different cohorts that have graduated from this school. The methodology involved the exploration of the existing documentation in archives, from the founding ideology up until today. The agreements that defined the graduate profile and that identify the architects trained at UBB, and the creation of the plans of study and the curriculum diagrams were also examined. The career paths of some of the teachers who brought to life these teaching processes in the classroom were analyzed in accordance with the national and local events of that time. Precisely when this school celebrates 50 years of training professional architects of great national prestige, this tour of its pedagogical models reaffirms its well-deserved third place ranking in architectural degrees in the country and revalidates its international accreditation by the Royal Institute of British Architects (RIBA) since 1991.
Palabras claves

Formación profesional, arquitectura, planes de estudios, programas, Bío-Bío
Palavras-chaves

Formação profissional; arquitetura; planos de estudos, programas, Bío-Bío
Keywords

Professional education, architecture, plans of study, programs, Bío-Bío 
Al cumplirse cincuenta años de la creación de la carrera de Arquitectura en la Universidad del Bío-Bío (UBB), resulta pertinente presentar este panorama que da cuenta de las propuestas aplicadas por la Escuela para la formación de sus profesionales. Sobre todo, a través de la comprensión del ideario de su creación, de los distintos planes de estudios y sus modificaciones y de la influencia que, en ellos, tuvieron los acontecimientos nacionales y locales.

1 | La Comisión Nacional de Acreditación (CNA) define un plan de estudios como "una explicitación del proceso formativo conducente al perfil de egreso, describiendo los contenidos (actitudes, conocimiento y habilidades), métodos pedagógicos (métodos y formas de enseñanza y de evaluación) y estructura curricular la secuencia sistemática e integrada de actividades académicas] de la carrera o programa de pregrado" (CNA, s/f., p. 17).

2 Roberto Goycoolea Infante, Osvaldo Cáceres González, Victor Lobos Lápera, Alejandro Rodríguez Urzúa, Augusto Iglesias Barrios, Alejandro Durán y Pedro Tagle.

\section{La carrera de Arquitectura}

Creada el año 1969, la carrera contó con el apoyo de la Delegación Regional del Colegio de Arquitectos de Chile y con el claro objetivo de descentralizar la enseñanza de la arquitectura que, por entonces, se dictaba exclusivamente en Santiago y en Valparaíso. Así, inició formalmente sus actividades el $1^{\circ}$ de junio de ese año bajo el alero de la sede Concepción de la Universidad Técnica de Estado (UTE) -devenida luego en la actual Universidad de Santiago (USACH)- después que fuera aprobada por "el Consejo Universitario de la sede penquista de la UTE... en su sesión $\mathrm{N}^{\circ}$ 495, del 9 de mayo de 1969, con el mandato de iniciar las clases ese mismo año" (Goycoolea Infante, 2017, p. 68). A lo largo de los cincuenta años de existencia de la carrera de arquitectura de la UBB, ocho fueron los planes de estudios ${ }^{1}$ que orientaron la formación de sus arquitectos.

Entre las primeras propuestas curriculares no se descubre una declaración formal del perfil de egreso; sin embargo, desde aquel primer plan de estudios hasta el vigente se detectan continuidades de importancia. La vocación regional es una de las que trascendiera, entendida en un principio como la necesidad de formar arquitectos que consideraran en sus proyectos las características climatológicas y culturales de la zona de Concepción y del sur del país, tales como el particular comportamiento del terreno ante los terremotos, la lluvia, la humedad la necesidad de ventilación, la vida interior etc. (Ibidem, p. 59).

En ese contexto, la revista $A U C A$ publicaba una reseña denominada "Nueva Escuela de Arquitectura: Concepción" para dar cuenta de su creación y, si bien lo relatado alli no puede ser considerado como el perfil del arquitecto que se pretendia formar, algunos extractos de aquel texto identifican el ideario de sus fundadores, el sentido que se quiso dar a esta nueva carrera de Arquitectura y la orientación que distinguiría su accionar y que perdura hasta el día de hoy:
Esta nueva Escuela tiene ante si toda una problemática ligada al
hábitat de su región, que alli se proyectan sin eludir el planteamiento
de los fenómenos universales. Se encuentra ubicada en un medio
industrial de gran potencialidad que hará posible la dialéctica
universidad-producción, clave de desarrollo. (Shapira, 1969, p. 4)

Con ello se dejaba claro que los arquitectos fundadores ${ }^{2}$ de la Escuela tenían claras las características del arquitecto por formar y así se manifestó en los planes de estudios de 1969 y en el del siguiente año.

Para la formulación de este primer plan de estudios (1969) fue clave la experiencia formativa que habia tenido el equipo fundacional. En su mayoría, eran egresados de la Universidad de Chile (UCh), educados con la malla renovada de 1945, misma que sirvió de guía para trazar el primero de los planes que implementarian en la escuela penquista (Goycoolea Infante, 2017, p. 69).

No obstante, las actividades académicas arrancaron con un plan de emergencia dado que el Consejo Universitario que aprobó la creación de la carrera condicionara el inicio del programa ese mismo año y su formalización se desprendería del Decreto ${ }^{3}$ que expresaba: "1. Créase en la Universidad Técnica del Estado la Especialidad de Arquitectura, dependiente de la escuela Industrial de Concepción, cuyo primer año comenzará a funcionar a partir del $1^{\circ}$ de junio de 1969." Dicho plan consideró solo asignaturas para el primer y segundo semestre y, durante ese lapso, el equipo docente estructuró el siguiente plan que contemplaba la totalidad de la formación, pero que recién se implementaría en 1970. 
Este primer plan consignaba solamente un año de estudios, no consideraba el sistema de créditos, sino de horas presenciales destinadas a cada asignatura de manera que el taller tenía dos grupos de estudiantes: para el primer y segundo semestre el total de carga de trabajo anual era de 70 horas repartidas en el 42,9\% para asignaturas relacionadas con el diseño: taller de diseño arquitectónico y geometría descriptiva, un 51,4\% destinado al área tecnológica con matemática, tecnología y física y un $5,7 \%$ para ciencias sociales. La fuerte carga en el área tecnológica en los inicios reflejaba la influencia de plan santiaguino de la UCh y, también, se vio influenciado por las capacidades de profesionales disponibles que debían afrontar la docencia en esas áreas del conocimiento. Y, aunque los programas de las asignaturas de ese primer plan no alcanzaron a ser formulados, las clases, las orientaciones y los contenidos mínimos fueron acordados por los profesores, en especial aquellos enfocados a la especialidad de la arquitectura.

Bajo estas condiciones, ingresó la primera ${ }^{4}$ cohorte de estudiantes a la carrera de Arquitectura en la entonces Universidad Técnica del Estado (UTE). La primera selección fue de 41 estudiantes; con posterioridad, se incorporaron 13 más, dando un total de 54 alumnos y alumnas iniciando los cursos un $1^{\circ}$ de junio de 1969. Asimismo, el primer año de las actividades docentes fue complejo, pues implicó trabajar en un plan concreto y en la orgánica que sustentaría el funcionamiento de la carrera oficialmente. Como resultado de este proceso, fue creado el "Departamento de Arquitectura", conducido por el arquitecto Víctor Lobos Lápera.

En el primer año de funcionamiento fue difícil establecer el grado de exigencia y de complejidad de tareas y proyectos que abordaban los talleres. Es que, junto con la novedad de lo que se desarrollaba aulas adentro, los alumnos esgrimieron por entonces una sobrecarga de trabajo y solicitaron postergaciones para sus entregas. Esos aplazamientos no fueron considerados $y$, consecuentemente, fue generada una crisis que motivaron el primer paro estudiantil y toma de la Escuela, la que provocó la anulación del primer año y llegó a tal punto que fue puesta en tela de juicio la continuidad de la carrera. Esa crisis convocaría un claustro de profesores en el cual participaron delegados de los estudiantes y, gracias a ello, se inició el análisis de los programas que sentarian las bases del segundo plan de estudios, el de 1970.

Coincidentemente, ese año marca un hito en la tradición de la Escuela de Arquitectura de la UBB, ya que se consumó el traslado de la sede al edificio que sigue ocupando y que, hasta entonces, pertenecía a la Escuela Técnica Industrial de la UTE (Figura 1).

\section{Plan de estudios de 1970}

En el documento elaborado por el Director de la Escuela de Arquitectura, Boris Aptecar González, se relata el tiempo transcurrido entre los tres primeros planes de estudios de la carrera -de 1969, 1970 y 1974-, denominándolo como el periodo de formación

$$
\begin{aligned}
& \text { durante el cual se implementa el plan de estudios [...] y se expande } \\
& \text { su área docente. En la fase final de este periodo se reformula el Plan } \\
& \text { de estudios original (planes 1969/1970) y se pone en marcha uno en } \\
& \text { función de las demandas de profesionales del entorno (plan 1974). } \\
& \text { Durante este periodo la función básica que se desarrolla es la de la } \\
& \text { enseñanza. (1984, pp. 2-3) }
\end{aligned}
$$

Así, el plan de 1970 surgía más completo e involucraba toda la trayectoria formativa que conducia al título profesional de arquitecto(a). Tenía una duración de 5 años o 10 semestres y en su "Memoria Explicativa del Plan de estudios de Arquitectura" dejaba establecidos los niveles formativos:

A) Formación Básica. En los niveles 100 y 200 se imparten las materias o cursos indispensables para la formación básica del arquitecto", correspondiendo al primer y segundo año de la carrera;

B) Formación Profesional. Se imparte en los niveles 300, 400 y 500, dando
4 | Previo al ingreso de esta cohorte, los alumnos debieron aprobar un examen de "Conocimientos especificos" que fue especialmente diseñado por los profesores de la nueva escuela. 
5 | Esta tradición tuvo que ser suspendida a partir del golpe militar de 1973 y fue retomada en el segundo semestre de 1991 gracias a un protocolo de permiso de trabajo, establecido en el Acuerdo N³ del "Acta Consejo de Escuela de Arquitectura" de su sesión №2 del 16 de junio de 1991.

6 | Eduardo Meissner fue cirujano dentista de profesión, además de licenciado en arte, artista, pintor y escritor; dueño de una cultura inquieta $y$ creativa, su presencia fue relevante en una carrera que daba sus primeros pasos en la formación de arquitectos.

\section{énfasis en el último nivel a la iniciación de especialización de acuerdo a vocación y tendencias del alumno, correspondiendo dichos niveles al tercer, cuarto y quinto año. (Lobos Lápera, s./f., pp.1-2)}

En este mismo documento, en su punto D), se referenciaban los motivos del ajuste del plan con el fin de responder las observaciones hechas por el Honorable Consejo Universitario en su sesión N523 del 19 de mayo de 1970, comentaban que,

$$
\begin{aligned}
& \text { tanto la Dirección del Departamento de Arquitectura, como la Comisión } \\
& \text { de Docencia del departamento (integrado por profesorado de todas } \\
& \text { las lineas docentes y con permanente representación estudiantil) } \\
& \text { se abocaron al reestudio del Plan considerando todos los niveles } \\
& \text { de la carrera y la experiencia obtenida en los primeros dos años de } \\
& \text { funcionamiento. (Ibídem) }
\end{aligned}
$$

El plan de estudios de 1970 mantiene asi cierta continuidad con el anterior de 1969, con un predominio en la formación de los aspectos técnicos de la arquitectura. Si se revisa la carga de trabajo de las distintas áreas formativas, el área de diseño arquitectónico/urbano y las asignaturas relacionadas comprendía el $34,6 \%$, el área técnica que involucraba aquellas asignaturas como edificación, estructuras o instalaciones, entre otras, disponía de un $45,6 \%$ de los créditos asignados y, con menor peso, figuraba el área de las ciencias sociales y de urbanismo, con un $15,7 \%$, y el de historia, con un 3,9\%.

Al término del quinto año el estudiante egresaba y disponía de 3 años para efectuar las restantes actividades curriculares que, aunque exigidas para la obtención del titulo profesional, quedaban fuera del plan. Para ello, se estimaba un mínimo 300 horas a repartir entre las asignaturas de Seminario y Práctica Profesional y otras 300 más para el desarrollo del Proyecto y Tesis de Título. Esta modalidad, muy común en las universidades chilenas de esos años, determinó que las tasas de titulación superaran con creces los años formales de duración de la carrera, llevando a que muchos estudiantes no cumplieran con estos requisitos y que tampoco obtuvieran el título profesional correspondiente.

Durante el periodo de vigencia del plan 1970 se desarrollaron también experiencias pedagógicas interesantes. Entre ellas, en 1972 y para optimizar los recursos docentes disponibles en función de la cantidad de estudiantes -bastante reducida en cuarto y quinto año- se implementaron talleres verticales. Esta innovación pedagógica no se mantuvo, básicamente por la dificultad de evaluar objetivamente los distintos niveles dentro de un mismo taller. También ese año, y por primera vez, se autorizó la posibilidad de ocupar -en horarios nocturnos- los espacios de la escuela para la preparación de las entregas finales de taller, transformándose esta en una práctica tradicional ${ }^{5}$ que perdura hasta hoy (Goycoolea Infante, 2017, p. 99).

Por esos años, la carrera ya disponia de un staff con 24 profesores que dictaban las asignaturas del plan de estudios, entre ellos destacan las figuras de Eduardo Meissner Grebe ${ }^{6}$ y de Rodulfo Oyarzun Philippi, pilares en los que se sostuvo la carrera.

El profesor Meissner estructuró la línea de "Configuración espacial", implementando una novedosa modalidad docente basada en equipos de trabajo. Así, las clases teóricas eran lideradas por él, mientras que el trabajo práctico de aplicación con los estudiantes y en los talleres era llevado a cabo por un equipo docente que se fue especializando en esta línea. Por largos años, la formación plástica de sus estudiantes fue una de las fortalezas que distinguiria a la Escuela de Arquitectura. El trabajo sistemático de este equipo de profesores fue creando y consolidando el ambiente de estudio y dio lugar a investigaciones que se expresaron en textos, entre los que se cuenta $\mathrm{La}$ configuración espacial sobre estructuras configuradoras y espacios configurados (Meissner, 1984), una versión en dos tomos que sistematizaba e ilustraba los contenidos teóricos con ejercicios desarrollados por los mismos estudiantes en clase. Desde 1970, los cuestionamientos globales hacia el Movimiento Moderno generaban ámbitos de discusión e involucrarian conceptos nuevos como la semiótica, el signo o la significancia; en esta línea, fue incorporado al plan de 1991 un curso electivo de quinto año, "Semiótica Arquitectónica" que estuvo a 

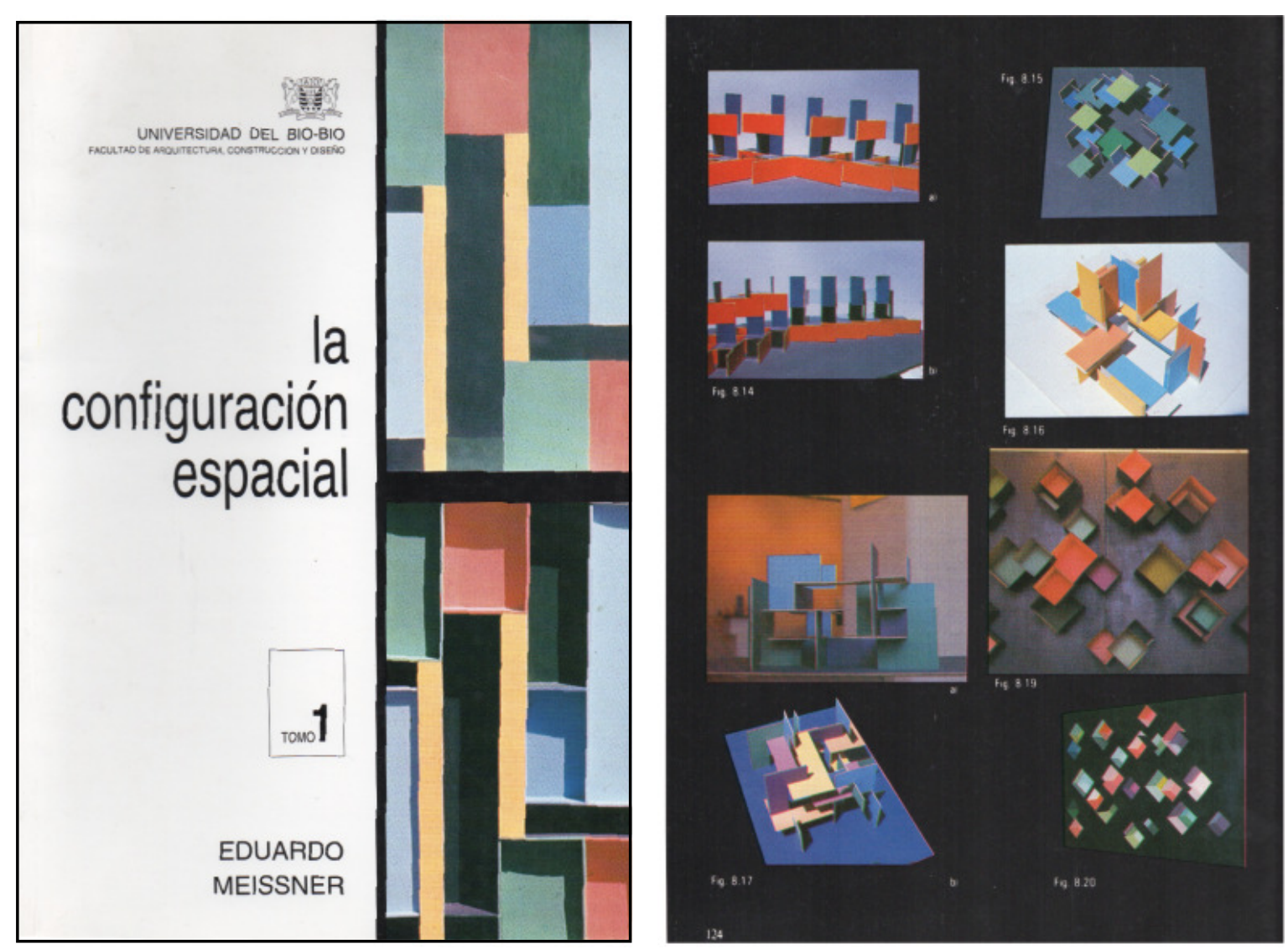

Figura 2. Der. Portada del Tomo 1 del libro de Meissner (1984) y ejemplos de ejercicios de formas y ambientación cromática (Meissner, 1984, tomo 2, p. 124).

cargo del profesor Jorge Harris Jorquera y que, Eduardo Meissner, ampliaría en su Teoría del signo en Arquitectura (Charlas de Nottingham) (2003), fortaleciendo de este modo la tendencia editorial disciplinar que caracteriza a la Facultad de Arquitectura, Construcción y Diseño (FARCODI) (Figura 2).

Otra figura relevante fue la de Rodulfo Oyarzun Philippi', incorporado en 1971 al equipo de profesores de la Escuela y a quien se le reconoce la estructuración de la línea de Historia de la Arquitectura en el plan de estudios de 1970. Su especial vocación por la enseñanza se expresaba en el vínculo con el espíritu humano, social y estético que, a su juicio, debía impregnar la acción del arquitecto en el ejercicio de su profesión. Esa sensibilidad se percibe en uno de sus textos publicado en E/Mercurio el 3 de mayo de 1931, al referirse al profesor Karl Brunner:

$$
\begin{aligned}
& \text { Necesitamos arquitectos que, tomando por base las ciudades } \\
& \text { existentes, sepan mejorar la composición plástica de ellas, sin perder } \\
& \text { de vista las eventualidades que el futuro pueda traernos, deben ser } \\
& \text { profesionales capaces de sentir el gran problema estético de la ciudad. } \\
& \text { Necesitamos arquitectos paisajistas que contribuyan a embellecer y } \\
& \text { alegrarnuestros pueblos, nuestras urbes, fundiéndolas conla naturaleza } \\
& \text { y arrancándolos, siempre más y más, del aislamiento, respecto a ella, se } \\
& \text { encuentran. }
\end{aligned}
$$

Sus ideas fueron, en efecto, reconocidas cuando recibió el Premio Nacional de Arquitectura (1974): Don Rodulfo... transmitió sin vacilar su preocupación por la problemática urbana y la responsabilidad social que le compete a los arquitectos, urbanistas y planificadores... (Muñoz, 2000, p.70). En su recuerdo y valoración de aquellos aportes, la Escuela de Arquitectura instituyó el Premio "Arquitecto Rodulfo Oyarzun Philippi" que distingue el mejor proyecto de título de la cohorte de estudiantes que, anualmente recibe su título profesional de Arquitecto(a).

7 | Arquitecto, urbanista, pintor, escultor, de importante trayectoria profesional y docente, participó en la fundación Instituto Nacional de Urbanismo (1929) del cual fuera su presidente, dictó la Cátedra de Urbanismo de la Escuela de Arquitectura de la UC (1930) y propuso al gobierno de la época la contratación del Dr. Karl Brunner Von Lehenstein para fortalecer la enseñanza del urbanismo en Chile y asesorar al Gobierno en temáticas urbanas. 
8 | Decreto №765, 17 de mayo de 1974, sede Concepción.

9 | Crédito facultativo era "toda actividad extra programática del alumno tendiente a su perfeccionamiento como profesional o al desarrollo de su personalidad" (Reglamento de Créditos Facultativos UTE, 1976).

\section{Plan de estudios 1973/1974}

Después de la interrupción de las actividades docentes, motivadas por el Golpe de Estado de 1973, se reiniciaron las clases el $1^{\circ}$ de octubre con un nuevo plan de estudios que fue oficializado por la UTE ${ }^{8}$. Este incorporaba a la malla 4 asignaturas de inglés y otras vinculadas al área de diseño, en lo que se refiere a expresión (con las asignaturas "Mano Alzada" y "Acuarela, Perspectiva y Topografía"). Al área de Historia se sumaron dos asignaturas de "Teoría de la Arquitectura"; en el área tecnológica se especificaron y detallaron otras explicitando el contenido en su denominación como "Estructuras Hiperestáticas", "Estructura Hormigón Armado", "Estructuras Metálicas y Madera", "Estructuras Especiales y Asísmicas", "Calor, Calefacción y Equipos Especiales" y "Tributación Contabilidad y Arancel”, entre otras.

Por primera vez se incluyeron los créditos de libre elección que tributaban a la formación personal y profesional del y la estudiante. Además del inglés añadido a la malla curricular, se incorporaron 40 créditos facultativos ${ }^{9}$ para "Ramos y Actividades Optativas"; iniciativa que se destacaba por la flexibilidad otorgada al plan de estudios, confiriéndole parte de la responsabilidad de su formación al mismo estudiante, máxime porque

\section{Estos créditos tienden a valorizar e incentivar académicamente las actividades de formación del alumno según su iniciativa particular o aconsejado por su tutor. Todo alumno de una carrera profesional debe ampliar los conocimientos específicos tendiendo a formarse una mente abierta a la cultura contemporánea y una comprensión de la historia.}

(Goycoolea Infante y Lobos Lápera, 1976, p.)

Igualmente, se mantenían los cinco años de duración propuestos en el plan de 1970 y la condición de egreso al $5^{\circ}$ año con la obligación de desarrollar las Prácticas de Diseño y las de Edificación, de 30 horas cada una, los denominados Créditos Especiales (fuera de la malla) y 100 créditos para la Tesis de Grado para optar al título profesional.

Con este nuevo plan de estudios, el Departamento de Arquitectura generó una suerte de orgánica de apoyo que le permitía coordinar los requerimientos de la carrera que iban en aumento. Surgió entonces la figura del Secretario Académico, asumido por Roberto Goycoolea Infante, en tanto Jorge Harris Jorquera se desempeñó como Coordinador Docente del Departamento. También se estructuraron una serie de normativas y de reglamentos fundamentales para el funcionamiento de la carrera y, al mencionado "Reglamento de Créditos Facultativos", se sumaron los Reglamentos de "Taller de Práctica Profesional", para la "Práctica de Diseño" y la "Práctica de Edificación", el de "Tesis de Grado", el de "Docencia del Departamento de Arquitectura" y el de "Estudio", además de detallarse todos los programas de las asignaturas que comprendía la carrera.

El Plan de estudios de 1973 consideraba en los dos primeros años una importante carga de trabajo para el estudiante pues cada semestre contemplaba ochos asignaturas, bajando a seis asignaturas en los siguientes $\left(5^{\circ}, 6^{\circ}\right.$ y $7^{\circ}$ semestre $)$; aumentaba luego en el $8^{\circ}$ y $9^{\circ}$ a siete, y finalizaba el $10^{\circ}$ con seis materias.

Si se analiza el peso de las distintas áreas formativas dentro de este plan, se constata una disminución en el total en los créditos relacionados con las áreas técnicas y de urbanismo y un incremento en las asignaturas relacionadas con las de diseño e historia. El 53,8\% de las asignaturas estaba relacionado con el área de diseño -un 19,2\% más respecto del plan de 1970; el área de las tecnologías disponía de un 36,6\% de los créditos totales -disminuyendo un 15\% en relación al anterior-; un 3\% de aumento figuraba para el área de historia y un 9,9\% de baja, para las de urbanismo y ciencias sociales. Aparecian, como se señaló, las asignaturas relacionadas con la formación integral del estudiante con 4 asignaturas de inglés que se incorporaban al plan con un 2,9\% del total de créditos. Esta tendencia de mantener en la formación de los estudiantes los aspectos y materias relacionadas con el desarrollo de las competencias proyectuales y de expresión arquitectónica -en desmedro de la parte técnica (estructura, edificación, instalaciones)se mantuvo como una constante en los siguientes planes de estudios 
implementados. Probablemente, esta situación obedezca a la incorporación de arquitectos recién titulados al plantel docente y, con ellos, una visión del ejercicio profesional un tanto diferente a la de los fundadores.

\section{Plan de estudios de 1979}

Este plan de estudios de 1979 se mantuvo hasta dos años después y, más que uno nuevo, se trató de un ajuste curricular al de 1973. Entre los aspectos más relevantes destaca la supresión de los cuatro cursos de inglés de la malla curricular y un leve incremento de 1,5\%, respecto del plan anterior, para los créditos vinculados al área de diseño arquitectónico y de expresión. El área de las tecnologías consideraba el 32,6\% del total de créditos (1,7\% más) y en las otras áreas como historia y urbanismo los incrementos fueron mínimos. Se mantuvo la duración de cinco años del plan de estudios con las actividades curriculares de Seminario, Tesis de Grado (título) y Prácticas de Diseño y de Edificación como requisitos para titulación, aunque no incorporadas al plan. También se mantuvo la obligatoriedad de cursar 40 créditos facultativos de libre elección y decisión por parte del o de la estudiante.

Sin embargo, la promulgación del DFL $N^{\circ} 1^{10}$ del MINEDUC afectaría gravemente a las universidades chilenas y, junto a ello, las inequidades hacia las universidades en regiones fue en crecimiento. Consecuentemente, fueron desarticuladas dos de las más grandes universidades estatales, como la UTE -afectando directamente a la carrera de Arquitectura en la sede Concepcióny la UCh, cuyas sedes regionales se transformaron en universidades derivadas $^{11}$, eliminándose toda participación estatal en el funcionamiento de todos los estamentos de estas instituciones. Esta ley implementó otros aspectos: por ejemplo, en su artículo 912, concedía a las universidades la exclusividad de otorgar los grados académicos de Licenciado, Magíster y/o Doctor, definiendo sus alcances y las exigencias para ser acreedores a ellos. El artículo $11^{\circ}$ concedía exclusividad a la universidad para "...otorgar los títulos profesionales respecto de los cuales la ley requiere haber obtenido previamente el grado de Licenciado en una disciplina determinada"; el $12^{\circ}$ definía las carreras consideradas "universitarias", entre las cuales se encontraba la de arquitectura, con facultad para otorgar el Título de Arquitecto(a) y el grado de Licenciado(a) en Arquitectura.

Así, la carrera de Arquitectura, en la sede Concepción de la UTE, contaba con estatus universitario y fue la oportunidad que facilitó la creación de la Universidad de Bío-Bío (UBB), en 1981. Como institución autónoma fue un desafío y una ocasión para definir sus objetivos, alcances e identidad como insitución regional, como también para reglamentar su orgánica de funcionamiento, normativa y régimen de estudios. En ese mismo año, se creaba la Facultad de Arquitectura (FA) bajo la figura de Escuela y de Departamentos. La Escuela (EA) albergaría la carrera de Arquitectura y sus primeros departamentos disciplinares fueron el de Diseño y Urbanismo, el de Patrimonio Cultural y el de Obras Civiles. Al siguiente año, con la creación de la Escuela de Construcción Civil, la Unidad pasó a llamarse "Facultad de Arquitectura y Construcción (FAC)": se designó como Decano al profesor Ricardo Hempel Holzapfel y como primer Director de Escuela al profesor Boris Aptecar González, en tanto que, el Director del antiguo Departamento de Arquitectura, el arquitecto Víctor Lobos Lápera, fue designado Rector de la Universidad.

\section{Plan de estudios 1982}

El decreto 63, del 29 de abril de 1982, y su modificación ${ }^{13}$ establecieron un régimen de estudios de carácter modular ${ }^{14}$ y el cumplimiento de determinados requisitos para la obtención de pregrado, grado académico o título profesional; esta planificación posicionaba a la UBB en un plano inédito a nivel nacional.

Fue el Vicerrector Académico, Giancarlo Roma, quien después de su perfeccionamiento académico en Estados Unidos, ideara este sistema
10 | A partir de este Decreto, promulgado el 30 de diciembre de 1980 y publicado el 3 de enero de 1981, el Estado inicia su desprendimiento de responsabilidad para financiar las universidades e inducirlas hacia el autofinanciamiento, incrementando la educación universitaria hacia el ámbito privado. Con esta regulación se eliminó el sistema de pago a las universidades vía arancel diferenciado y proporcional al ingreso económico de las familias, iniciando el sistema del Aporte Fiscal Indirecto.

$11 \mid$ Para acceder a esta derivación la universidad debía contar con una de las 12 carreras consideradas universitarias $\mathrm{o}$, en su defecto, se transformaria en instituto profesional.

\section{2 | Título III, Grados Académicos y Títulos Profesionales.}

$13 \mid$ El decreto 9, del 17 de enero de 1986.

14 | Entendiendo cada módulo como un conjunto estructurado de asignaturas según el programa. 
15 | La CNAP fue creada por Decreto Supremo 51, del 6 de abril de 1999, del Ministerio de Educación con la finalidad de establecer un sistema evaluador y con mecanismos de aseguramiento de la calidad en la Educación Superior en los programas de pregrado.

16 | Este tratado generó el Espacio Común Europeo de Enseñanza Superior y, en consecuencia, los diferentes proyectos que surgieron fueron contribuyendo a la implementación de tales acuerdos. Entre ellos, los proyectos Alfa Tuninig, en sus diferentes versiones, que permitieron levantar información relevante relacionada con las competencias transversales, indicando más demanda en el campo laboral, homologando las competencias disciplinares de las titulaciones, creando un sistema de créditos transferible y transitando desde un paradigma basado en conocimientos a otro basado en competencias o resultados de aprendizajes.

inspirándose en modelos allí conocidos. En términos globales, el método contemplaba -para una titulación de seis años-, tres módulos de formación de dos años cada uno: el Módulo Básico, el Medio y el Avanzado, cada uno con exigencias y complejidades crecientes. Cada módulo se cerraba luego de un acto administrativo, de manera que el estudiante accedia a la siguiente fase una vez que fueran completadas las asignaturas correspondientes y alcanzara el rendimiento ponderado exigido. El plazo de cierre máximo era de 4 años ya que las asignaturas aprobadas tenían esa vigencia, de no cerrarse el módulo correspondiente el(la) alumno(a) debía volver a cursar las asignaturas aunque estuvieran aprobadas con antelación. Otra innovación interesante de este régimen fue la escala de calificaciones aplicada a través de conceptos.

En general, aquellos planes de estudios no se concebian a partir de la definición de un determinado perfil de egreso -tal como la actual exigencia de la CNA-, sino que se diseñaban tomando como referencia los existentes elaborados por las universidades tradicionales. Tampoco existían orientaciones muy definidas en los aspectos curriculares universitarios por parte del MINEDUC, hasta la promulgación del DFL No1 antes citado. Por entonces tenían más injerencia y peso los Colegios Profesionales, quienes cautelaban la idoneidad de los procesos educativos conducentes a títulos profesionales que garantizaran el adecuado desempeño en el medio profesional.

Lo destacable es que para el diseño de este Plan de estudios de 1982, el equipo de profesores fue convocado a participar en jornadas de trabajo que permitieron acordar la definición del perfil de egreso. En la "Definición de las metas, objetivos y perfil de los Módulos Básico, Medio y Avanzado del programa de Arquitectura" (1982, p. 1), se determinó que el Arquitecto egresado de la UBB se identificaría por:

- Su capacidad de autogestionar su trabajo profesiona

- Su profundo conocimiento de las raíces regionales, debiendo incluso cuando ello sea necesario, poder establecer dichas raíces

- Reconocer el destino forestal y maderero de la región lo que implica un profundo manejo del diseño arquitectónico y de la tecnología de la madera.

Con ello quedaron establecidas las metas pedagógicas y de conocimiento que debía alcanzar el estudiante en cada uno de los módulos. Esta definición del perfil de egreso de la EA significó adelantarse unos 20 años a las futuras exigencias que imprimirian, en el diseño de los planes de estudio, el MINEDUC, la futura Comisión Nacional de Acreditación de Pregrado ${ }^{15}$ (CNAP) y la CNA (2006). En otras palabras, la carrera de Arquitectura de la UBB se anticipó en varios años a lo acordado en Bolonia en $1999^{16}$.

Otro aspecto importante fue definir el Módulo Medio, en el segundo ciclo formativo, como conducente al Grado Académico de Licenciado y que establecería los conocimientos y habilidades que debía alcanzar el estudiante para ser acreedor de este Grado Académico; la situación se daba al término del cuarto año, en el octavo semestre y se ha mantenido en los sucesivos planes de la carrera.

En 1982, con la implementación del nuevo plan modular de la carrera y para fortalecer la planta académica de jornada completa, se llamó a concurso docente. Fueron seleccionados entonces los profesores Jaime Garretón Risopatrón para el área de Urbanismo, Germán Escudero para el área de Estructuras y la nueva carrera de Construcción Civil, y quien esto escribe para el área de Diseño e Historia de la Arquitectura. Cabe recordar que desde 1981 se desempeñaba el profesor Hans Fox Timmling, quien tuvo un rol protagónico en iniciativas como la creación del Centro de Desarrollo en Arquitectura y Construcción (CEDAC), en la edición de la primera revista de la Facultad, Arquitecturas de/ Sur (1983), y en la promoción de la acreditación internacional.

Así, el Plan de estudios de 1982, además de adecuarse a la normativa del DFL №1, instrumentó en la malla asignaturas como "Metodología de la Investigación" y "Seminario" que permitían comprobar las capacidades y conocimientos para la obtención del grado de Licenciado en Arquitectura. Al respecto, el "Documento de las Metas, Objetivos...” antes citado, expresaba que: 

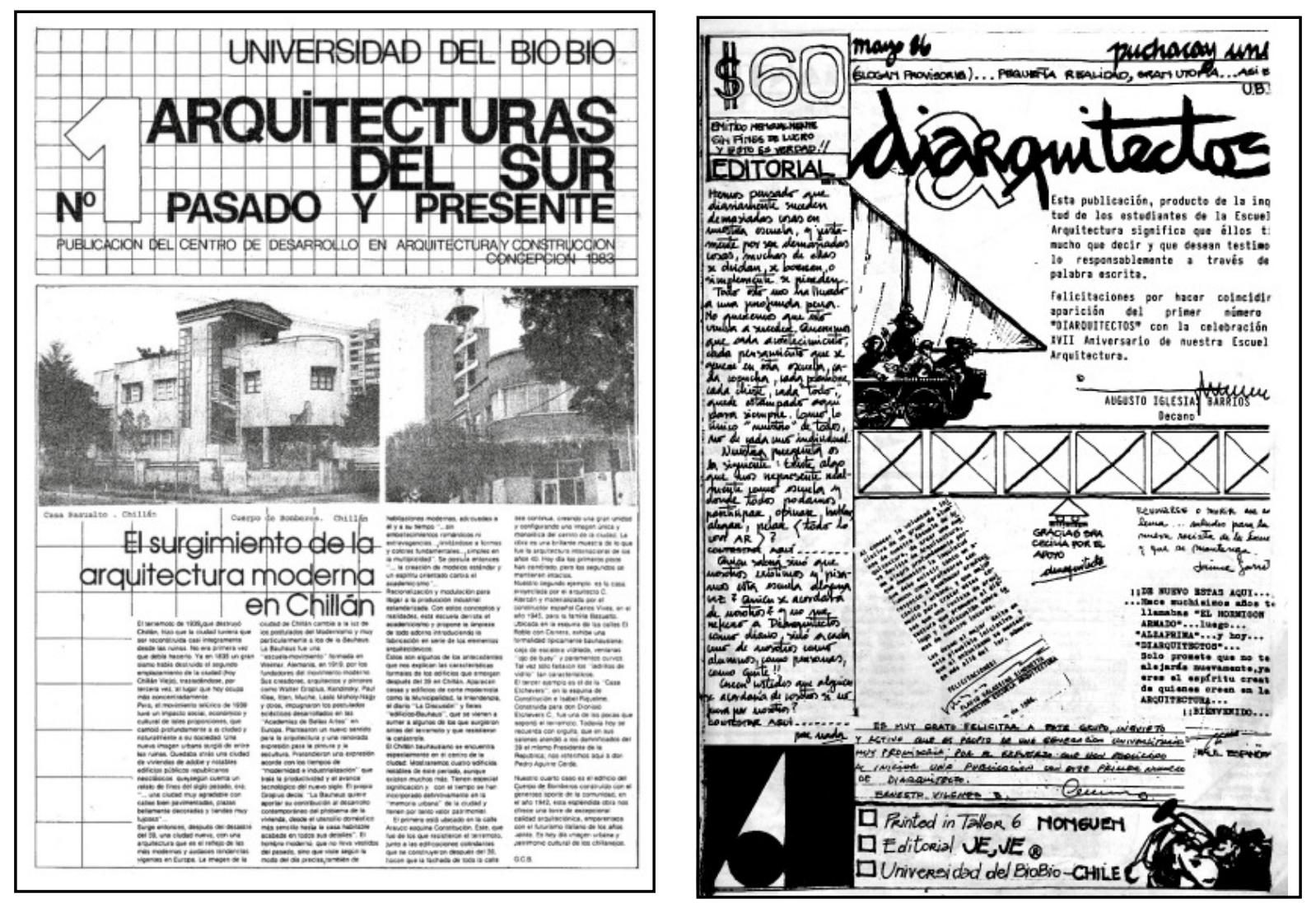

Figura 3. Portadas de las primeras ediciones de las revistas de la UBB Arquitecturas de/ Sur (junio, 1983) y Diarquitectos (mayo, 1986).

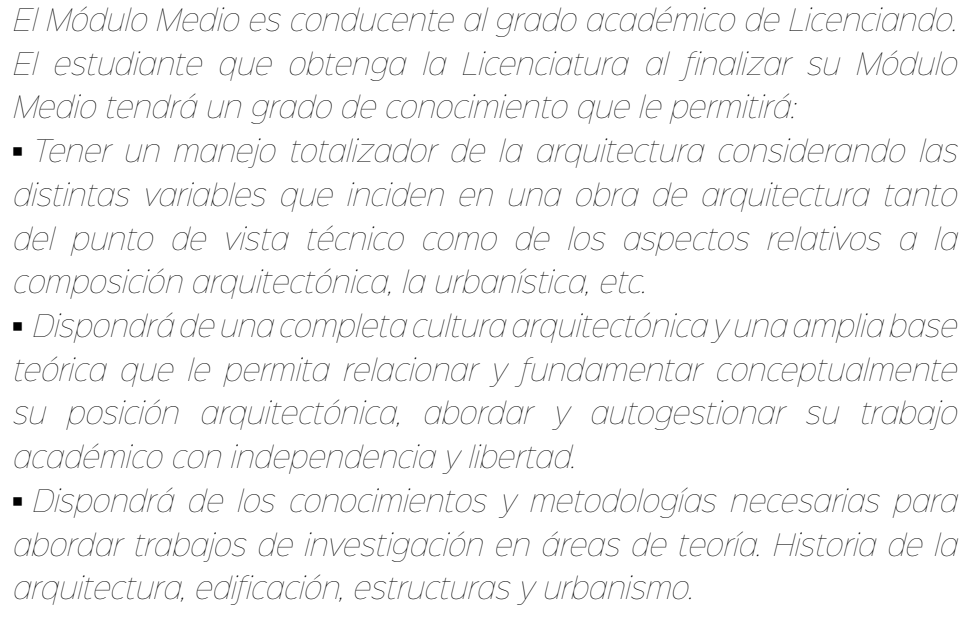

El plan de 1982 contemplaba 6 años de duración formal, como parte del proceso pedagógico incorporaba la Formación General que se materializaba en el Área IV de ese plan y tendió a mejorar las tasas de titulación ${ }^{17}$ gracias a la secuencia de Taller XI y Taller XII (título). Fue un plan semiflexible, que establecía un mínimo de créditos por área de conocimientos y que se cumplimentaban al cabo de cada Módulo; simultáneamente, el estudiante podía inscribir otros créditos de su elección y completar el total, considerando que comprendía 78 de libre configuración (12 disciplinares, 16 de inglés y 50 generales).

En 1988 y gracias a la fusión de la UBB (heredera de la UTE, sede Concepción) con el Instituto Profesional de Chillán (sede de la UCh), nacía la Universidad del Bío-Bío, fruto de la Ley №18.744, publicada el 29 de septiembre de 1988 en el Diario Oficial. Este acontecimiento implicó una revisión de todos los procesos y actividades de sendas instituciones. El ingeniero Guillermo Schaffeld Graniffo fue designado -por parte del gobierno central- como Rector delegado
17 Las cifras de titulación eran bajas dados los criterios de los planes anteriores, que permitian un lapso de tres años entre el egreso en el quinto año y la entrega del proyecto final de título. 
18 | La carrera de Diseño Gráfico había sido abierta en marzo de 1969, en Chillán, aún dependiente de la UC, y se adscribe a la Facultad como producto de la citada fusión. Ello determinó el cambio de nombre de la Facultad de Arquitectura Construcción y Diseño (FARCODI).

19 | FARCODl: ver nota ut supra.

$20 \mid$ El primero se editó el 22 de junio de 1990.

$21 \mid$ Del 24 de agosto de 1990. para concretar la fusión entre las dos instituciones. La orgánica implementada una estructura organizacional habitual en el ámbito de las ingenierias- afectaría directamente a la EA, debido a la supresión de las escuelas y, en consecuencia, la transformación en un gran departamento disciplinar o multidisciplinar que aglutinaba a todos los académicos afines e incorporaba, además, los programas de pregrado administrados por un Jefe(a) de Carrera, con dependencia directa del Director de Departamento. Esta nueva estructura implicó la supresión de la EA y de los departamentos disciplinares de la FAC, pero las carreras quedaron bajo el alero de los departamentos afines a su disciplina según tres nuevas unidades: el Departamento de Arquitectura (que comprendia las áreas de diseño, composición, representación, historia y urbanismo relacionados con la carrera de Arquitectura), el Departamento de Ciencias de la Construcción y el Departamento de Diseño, con sede en Chillán, donde se impartía la carrera de Diseño Gráfico ${ }^{18}$; asimismo la UBB, quedaría constituida en dos sedes, la de Concepción y la de Chillán, esta última con dos campus: La Castilla y Fernando May.

Entonces, fue designado como decano de la FARCODI ${ }^{19}$ el arquitecto Ricardo Hempel Hotzapel, como Director del Departamento de Arquitectura, quien esto escribe, y como Jefe de la Carrera de Arquitectura, el arquitecto Roberto Burdiles Allende. La principal tarea que emprendieron fue la de estructurar el funcionamiento de esta nueva orgánica, que se resumió en el documento "Organización y Estructura del Departamento de Arquitectura. Facultad de Arquitectura Construcción y Diseño de la Universidad del Bío-Bio" (2 de mayo de 1990). Allí se explicitaba la operatoria de la unidad en base a las grandes áreas de conocimiento de Diseño, de Historia y Teoría de la Arquitectura y de Urbanismo, quedando cada una a cargo de los arquitectos Roberto Goycoolea Infante, Héctor Gaete Feres y María Dolores Muñoz, respectivamente. Además, se designaron coordinadores de Departamento, para el área de construcción a Raúl Soto Castillo, para la de investigación y perfeccionamiento a Iván Cartes Siade y para la de extensión a Gonzalo Cerda Brintrup. A efectos de conocer los avances del Departamento de Arquitectura e involucrar a sus académicos y docentes, fue redactado un Boletín Informativo ${ }^{20}$, de frecuencia semanal y a cargo del arquitecto Carlos Jara Fernández, cuya edición facilitó informar y trazar el rumbo hacia la actual estructura orgánica que rige la Facultad y los procesos de rediseño de los planes de estudios de 1989 y de 1991 (Figura 4).

\section{El Plan de estudios del año 1989}

Tal como se mencionó, la fusión entre la UBB y el Instituto Profesional de Chillán implicó un nuevo Régimen de Estudio con impacto en la sede Concepción, en la que se disolvió el sistema modular que regía a las carreras alli dictadas desde el año 1982. Estas nuevas orientaciones emanadas por la Vicerrectoría Académica -a cargo de Soledad Ramírez Gatica- y desde la Dirección de Docencia -a cargo de Victor Estrada-, exigieron reformular rápidamente un nuevo plan de estudios para la carrera de Arquitectura, pues, institucionalmente, todas el resto iniciaban el año académico de 1989 bajo el nuevo modelo.

Entre los aspectos que dan cuenta de los cambios introducidos por aquella administración, figura la adopción de una nueva escala de calificaciones ( 0 a 100), la extensión del semestre académico a 16 semanas, la definición de número de créditos destinados a asignaturas de Formación Integral y que debian contener los planes de estudio de acuerdo a su duración, a la vez que una nueva codificación para cada asignatura. El Decreto $\mathrm{N}^{1} 636^{21}$ oficializó ese plan de estudios que se aplicó desde marzo de 1989; sin embargo, su práctica abarcó solo dos años y fue considerado como una transición hacia el plan de 1991.

Dicho plan, de 1989, contemplaba una duración de seis años, incorporaba en el sexto año -al igual que el plan anterior, modular- el "Taller de Diseño Arquitectónico XI" y el "Taller de Diseño Arquitectónico XII”, correspondientes al Proyecto de Título, siendo el primero para entregar el anteproyecto y el segundo de desarrollo y entrega final. Asimismo, la computación e informática sentaban presencia y fue necesaria su incorporación en la formación; para ello se incorporó 


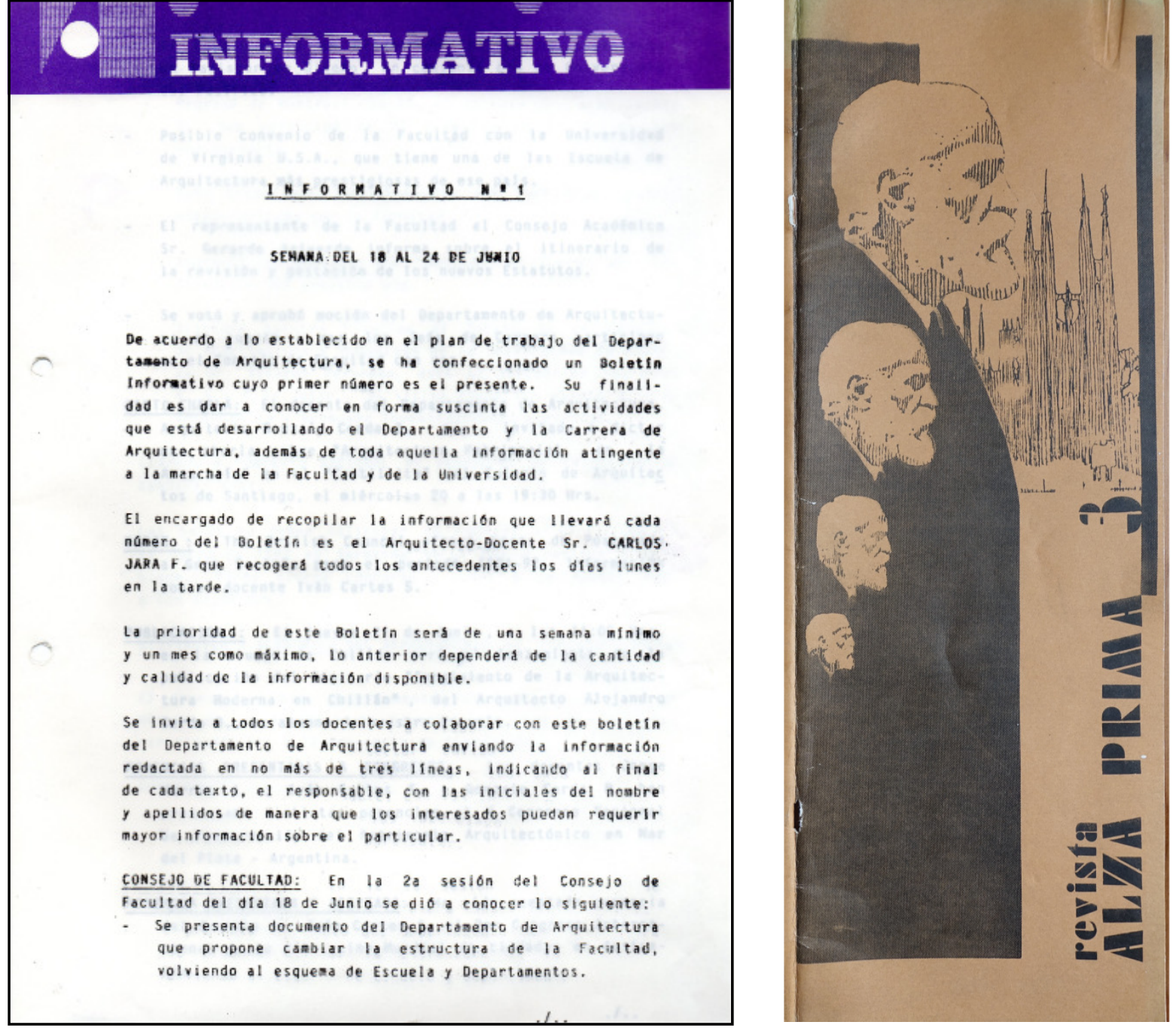

Figura 4. Boletín informativo de 22 de junio de 1990 y portada de la Revista Alza Prima (3), 1983.

en el tercer semestre de la carrera "Introducción al Diseño por Computación" y, en el noveno, "Diseño por Computación", conducidas por el arquitecto Rodrigo Garcia Alvarado. Las prácticas profesionales (que ocupaban unas 600 horas en total) fueron requisito para la obtención del título profesional, aunque no estaban incluidas directamente en el plan de estudios.

De esta manera, el organigrama contenía un total de 245 créditos, de los cuales un 52,2\% estaba relacionado con el área de diseño (taller, configuración espacial y expresión arquitectónica), incrementándose en un 4,7\% en relación con el plan de 1982; y un 28,6\%, con el área técnica (matemáticas, estructuras, edificación, instalaciones y programación), disminuyendo un 3,8\% respecto al plan anterior. Las áreas de urbanismo disponian de un $8,5 \%$ e historia con un 6,5\% del total, exhibiendo un leve crecimiento de 1,3\% y $1 \%$, respectivamente. La "Formación integral" - pese a la exigencia de considerar 5 asignaturas mínimas en el diseño del plan de estudios- se reducia un $2 \%$ en comparación con los 6,4\% del curriculum previo, aun considerando solo un crédito de carga directa semanal. Se suprimía también "Metodología de la investigación", dejándose exclusivamente "Seminario" para el décimo semestre.

Con el plan de 1989 se acentuó la tendencia que se vislumbraba en el plan de estudios de 1974, al recortarse, como entonces, los aspectos técnicoconstructivos en beneficio de los relacionados con la enseñanza del proyecto y con la capacidad de proyectar.

La llegada de la democracia al año siguiente promovió acontecimientos que marcarian el derrotero de la FARCODI y de la propia UBB. Así, el 3 de mayo de 1990, de acuerdo con la nueva estructura orgánica institucional, se constituyó el Consejo de la FARCODI, presidido inicialmente por el Rector y la Vicerrectora Académica. El fin del gobierno de facto también había puesto término al sistema de rectores delegados ${ }^{22} \mathrm{y}$, por primera vez en la Universidad, la votación del Rector surgió del sufragio de todos los académicos, eligiéndose en agosto de
22 | Designados hasta entonces por autoridades del gobierno central. 


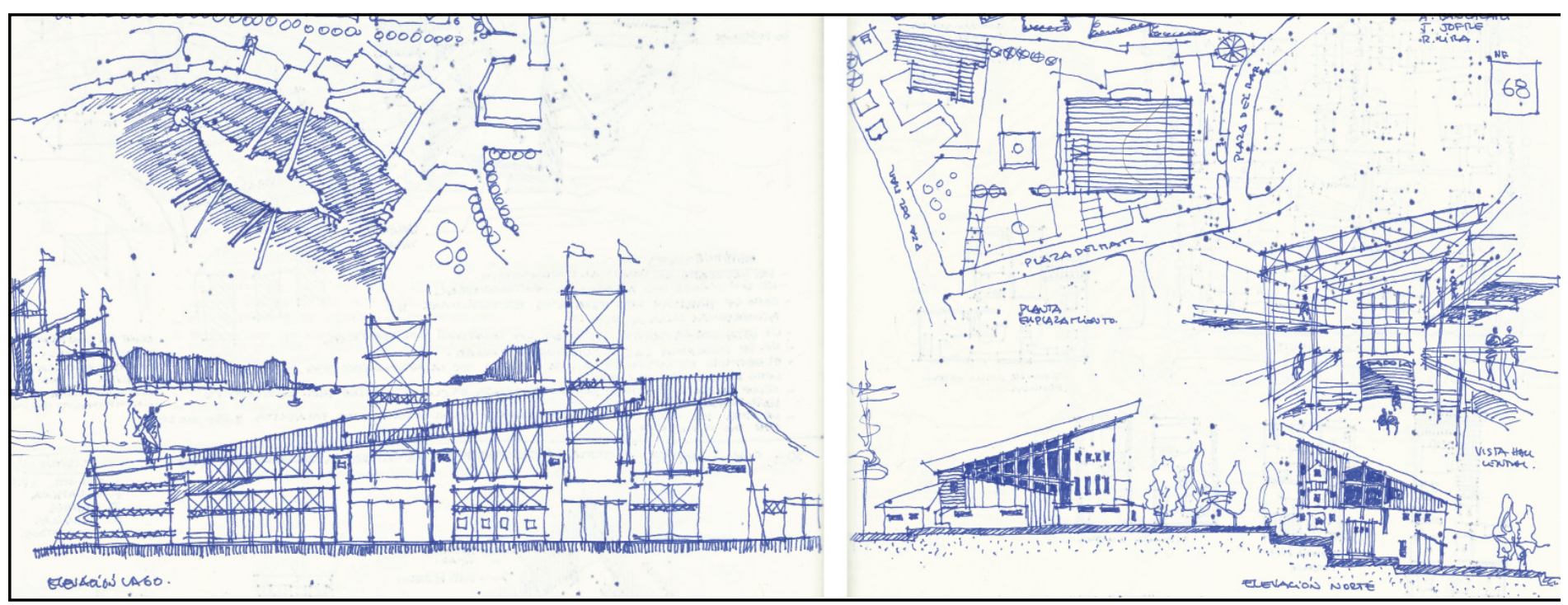

Figura 5. Apuntes de Proyecto Final de carrera tomados por el autor. De izq. a der:. "Club náutico Pucón" de Hugo Marquez (1997); "Equipamento comunitarios" de Juan Marcos Solis

23 | El RIBA es una organización de arquitectos del Reino Unido, fundada en 1834. En 1863, estableció un sistema de acreditación de las carreras de Arquitectura, que fue obligatorio a partir del año 1882 para todas las instituciones de educación superior de aquel país. La finalidad de la RIBA es introducir criterios de calidad en la enseñanza de la Arquitectura y evaluar la efectividad de los aprendizajes de los estudiantes.
1990, y por un periodo de cuatro años, al arquitecto Roberto Goycoolea Infante, quien sería reelecto en el período siguiente. De igual modo, en ese primer gobierno universitario democrático participaron otros académicos vinculados al Departamento Arquitectura, tales como Hilario Hernández Gurruchaga, como vicerrector académico, y Héctor Gaete Feres, como director de planificación.

Aquel Acta de constitución del Consejo de la FARCODI indicaba que "El Director del Departamento de Arquitectura da a conocer la aspiración de volver al esquema de Escuela, señalando problemas en el actual sistema, como el menoscabo de la función del Jefe de Carrera y la interferencia entre este y el Director de Departamento" (p. 3). Fue el turno entonces de comisionar al Director del Departamento para que coordinara los estudios conducentes a formular una nueva estructura orgánica de la Facultad bajo la modalidad de departamentos y de escuelas.

En mayo de 1990, gracias a las gestiones del académico Hans Fox Timmling, la Facultad recibió al arquitecto británico Christopher Riley, miembro del del Royal Institute of British Architects ${ }^{23}$ (RIBA). De este encuentro surgió la posibilidad de postular la carrera de Arquitectura de la UBB al proceso de acreditación internacional que promocionaba esa institución y se acordó entonces la visita del Visiting Bourd para noviembre de 1991. Hacia junio de ese año la FARCODI recibía al arquitecto Ricardo Beckerman, decano de la Facultad de Arquitectura de la Universidad de Mendoza (Argentina); una visita que concluyó con la firma del convenio bilateral de intercambio estudiantil y que permitió que, en estadías semanales, los alumnos de la EA desarrollaran sus proyectos finales en la institución trasandina y lo propio sucedía al semestre siguiente cuando alumnos argentinos acudían a las aulas penquistas. Este intercambio además de productivo, fue pionero para el sistema de movilidad estudiantil que se implementaría recién en la primera década de 2000 con el MEC (Programa Movilidad Estudiantil Nacional del Consorcio de Universidades Estatales).

Por otro lado, y a causa del impacto que tenía la aplicación del régimen 


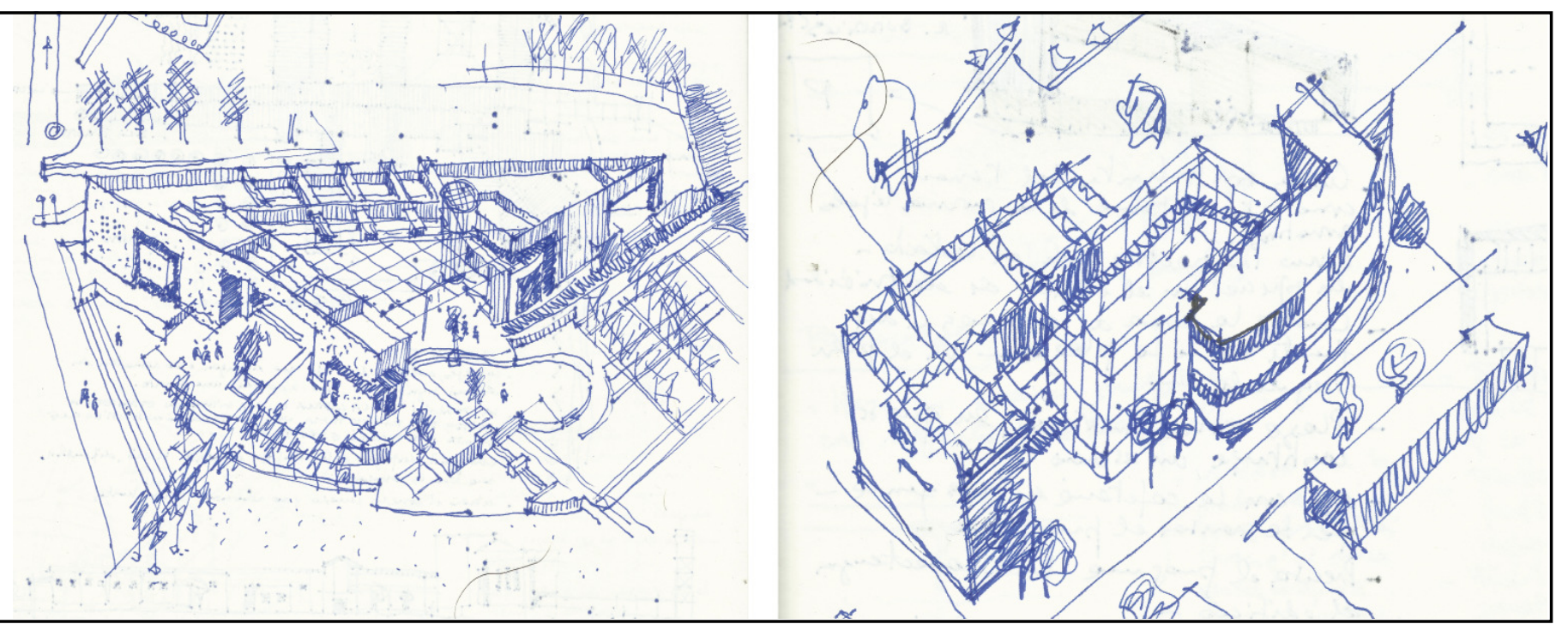

"Edificio consistorial para San Pedro de la Paz" de Fabiola Godoy (2000) y "Oficina municipal de la juventud" de Marcelo Pergola (2007).

de estudio institucional en el trabajo académico de los estudiantes, surgieron cuestionamientos por parte de profesores y alumnos. El documento elaborado por el director del Departamento de Arquitectura, titulado Segundas Jomadas de Estudio Crítico Entomo a la Enseñanza de la Arquitectura, daba cuenta de sesiones de trabajo de académicos del área de diseño realizadas entre el 26 y 27 de diciembre de 1989 , en la que se atendieron los puntos críticos por los que atravesaba la carrera. El análisis se enfocaba en tres aspectos: el perfil del arquitecto y su relación con el plan de estudios, la correspondencia entre enseñanza de la arquitectura y el régimen de estudios y la metodología de la enseñanza en los talleres de diseño arquitectónico. Los resultados de estos encuentros se compilaron en el documento "Factores de un sistema semestral que afectan el desarrollo del plan de estudios de Arquitectura"; un documento que sintetizaba los problemas del proceso formativo de los estudiantes y las intenciones de resolver las causantes de esta crisis que los afectaba directamente en su rendimiento académico. Por lo mismo fueron convocadas las Primeras jornadas -desarrolladas entre el 26 y el 29 de septiembre de 1990-, que contaron con la participación del Centro de Estudiantes de la carrera y congregó académicos, docentes y expertos, entre los cuales destacan Gustavo Munizaga Vigili ${ }^{24}$ y María Ángela Schweitzer Lopeteguii ${ }^{25}$, todos los cuales debatieron las dificultades detectadas en el funcionamiento de la Escuela.

Una siguiente reunión, Encuentro interno de la escuela de Arquitectura (14 y 15 noviembre), daba continuidad a la anterior, reuniendo otra vez al total de la comunidad de Arquitectura. Funcionó con mesas de trabajo en distintos temas, coordinadas por un profesor y, al finalizar, cada una exponía sus conclusiones. La Dirección del Departamento de Arquitectura presentó una propuesta de creación de un nuevo plan de estudios con un régimen distinto al institucional, adecuado ahora a las características propias de la enseñanza disciplinar y con énfasis en la incompatibilidad entre los tiempos de los procesos creativos y los destinados a las asignaturas tradicionales que repercutian en las evaluaciones
24 | Había obtenido su Máster en Arquitectura y Diseño Urbano en la Universidad de Harvard, siendo uno de los primeros en alcanzar este grado académico $y$, por entonces, era profesor de la Escuela de Arquitectura de la UC y de la Pontifica Universidad Católica (PUC).

25 Se trata de una de las primeras académicas en realizar investigaciones sistemáticas relacionadas con la formación de arquitectos en las universidades del país, como dan cuenta sus textos "La Formación del arquitecto: Perspectivas" (1986) e "Iniciación profesional y Formación Universitaria. Casos de Arquitectos titulados en la Universidad Católica de Chile" (1989), editados por la Corporación de Promoción Universitaria. 
finales de los ramos teóricos y la de los talleres. Así, se propuso generar una estructura que permitiera diferir los momentos de evaluación entre estas áreas $y$, consecuentemente, surgió aquella que caracterizó al Plan de estudios de 1991.

Resulta relevante advertir que, en la concepción de este nuevo plan de estudios, el proceso fue consensuado gracias a la amplia participación de los estamentos de la Facultad quienes aportaron el diagnóstico y ofrecieron soluciones a los inconvenientes que afectaban la marcha de la carrera. Aunque con plazos acotados, el Departamento de Arquitectura y la Jefatura de la carrera se abocaron a la tarea de diseñar el nuevo plan de estudios y confeccionar los programas de asignaturas que debian presentarse en enero de 1991, aguardar la aprobación por el Consejo Académico e iniciar su implementación a partir de marzo de ese mismo año.

La definición del perfil de egreso que estructuraria el plan de estudios de 1991 se basó en la "Definición de los Módulos Básico, Medio y Avanzado y la Licenciatura del programa de Arquitectura" (Valassina Simonetta, 1987, p. 1), donde establecía que

El campo profesional del arquitecto como aquel consagrado por la sociedad y establecido por las normas legales vigentes que en general comprende:

- La proyectación arquitectónica y urbana

- La organización, gestión, administración de proyectos y supervisión de obras arquitectónicas y urbanas.

- Las actividades académicas (docencia, investigación y extensión)

Asi el Perfil del Arquitecto de la Universidad del Bío-Bío identificaba cuatro características:

1. Su profundo conocimiento de las raíces y el medio ambiente regional y ser capaz de establecer aquellos sistemas necesarios para acoger en mejores condiciones el quehacer de la sociedad;

2. Reconocer el destino forestal maderero e industrial de la región, lo que implica un profundo manejo del diseño arquitectónico, urbano y de la tecnología en la edificación especialmente referida a la madera;

3. Su capacidad de autogestionar su trabajo profesional y

4. Sólida formación ética, humana y profesional.

En las "Segundas Jornadas de estudio crítico en torno a la enseñanza de la arquitectura", realizadas el 26 y 27 de diciembre de 1989, se efectuaron algunos ajustes, luego reunidos en su documento final: la frase "Sólida formación ética, humana y profesiona” fue reemplazada por la de fomentar "Una orientación hacia la autoformación y permanente perfeccionamiento profesional”; en igual sentido, se aclaró que en la formación del arquitecto UBB debia existir el convencimiento de que la arquitectura "es obra construible y habitable" y no solo "obra proyectada" (1989, pp. 3-4).

\section{El Plan de estudios de 1991}

La efectiva participación de la comunidad de Arquitectura en las Jornadas de fines de 1989, permitieron rápidamente la implementación de este nuevo plan. Se trataba de un plan de estudios anual, con organización trimestral, atributo que lo posicionó como innovador, tanto en su diseño como en su concepción.

Entre las premisas que determinaron su construcción pueden citarse:

- La constante nivelación de los estudiantes en la malla curricular, que alentó la concordancia entre asignaturas teóricas y el Taller de Diseño Arquitectónico.

- La continuidad de los procesos de enseñanza del Taller de Diseño Arquitectónico, lo cual evitó coincidencias en los periodos de evaluación con las asignaturas teóricas.

- La existencia de un espacio de dedicación exclusiva al taller, que facilitó la entrega de proyectos, presentaciones a comisiones y evaluaciones del proceso de diseño.

- La permisión a los profesores de las asignaturas teóricas y prácticas -distintas al taller- para apoyar el trabajo estudiantil en la fase final de proyectos.

El Consejo Académico de la UBB, en sesión ordinaria, el 27 de febrero de 

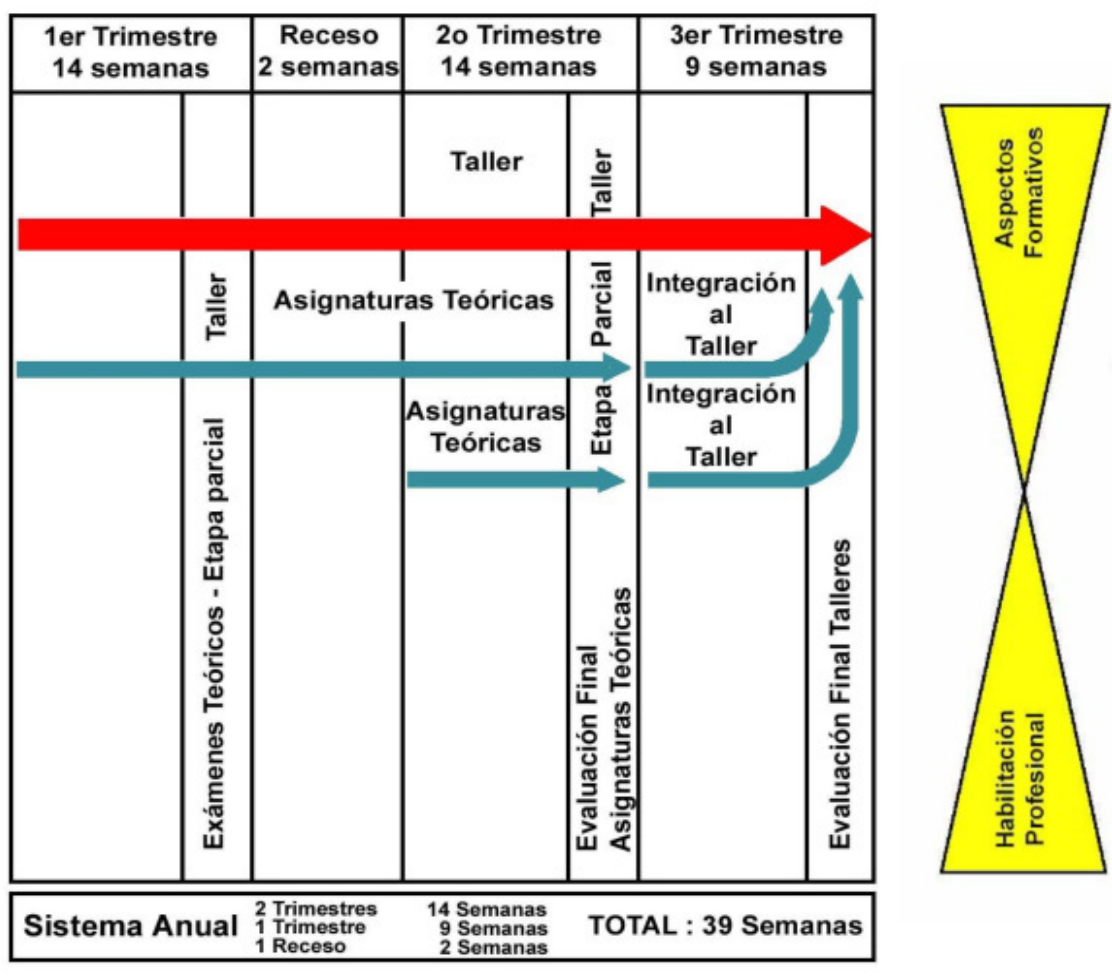

CICLO INICIAL - FORMATIVO SELECTIVO

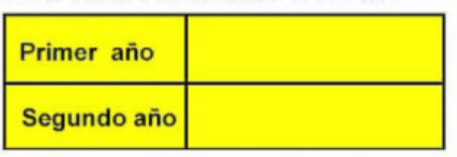

CICLO INTERMEDIO - FORMATIVO INSTRUCTIVO

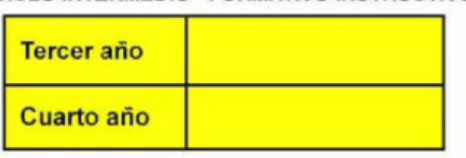

CICLO FINAL - HABILITACION PROFESIONAL

\begin{tabular}{|l|c|l}
\hline \multirow{2}{*}{ Quinto año } & Seminario Taller & $\begin{array}{l}\text { LICENCIATURA EN } \\
\text { ARQUITECTURA }\end{array}$ \\
\cline { 2 - 2 } & $\begin{array}{c}\text { Prácticas } \\
\text { Profesionales }\end{array}$ & \\
\hline Sexto año & Proyecto de Título & $\begin{array}{l}\text { TITULO } \\
\text { PROFESIONAL }\end{array}$ \\
\hline
\end{tabular}

Figura 6. Estructura general (dir.) y esquema de ciclos formativos (isq.) del plan de estudios de 1991 de la carrera de Arquitectura. Fuente: Archivo FARCODI-UBB.

1991 aprobó el nuevo plan de estudios de la carrera de Arquitectura y facilitó su aplicación al inicio de ese mismo año académico. En la misma sesión se aprobó la nueva estructura de la FARCODI en base a departamentos, unidades encargadas del desarrollo de las disciplinas asociadas, a escuelas encargadas de la administración de los planes de estudios y de la trayectoria formativa de sus respectivos estudiantes. De esta manera, la carrera de arquitectura recuperaba la Escuela como el ámbito que le daba identidad (Figura 5).

En esencia, el Plan de estudios de 1991 preveía una duración de seis años, no se estructuraba en base a créditos, si no a horas pedagógicas ${ }^{26} y$, aunque el balance con los planes anteriores -que ponderaban el sistema de créditos- puede ser impreciso, algunas cifras de análisis resultan interesantes. Este nuevo organigrama contemplaba 5782 horas y, en términos porcentuales, el $65,4 \%$ se vinculaba al área de diseño, mostrando un incremento sustancial del 13,2\% en comparación al plan de 1989 que disponía solo de un 52,2\% de créditos destinados a esta misma área. Las horas restantes también reducian su porcentaje de participación; por ejemplo, las técnicas disponian de 16,7\% de tiempo frente al 28,6\% con el que contaban previamente, decreciendo un 11,9\%; ocurría lo mismo con las áreas de historia, que bajaban un 2,1\%, y con las de urbanismo que lo hacian un 3\%. Por contraparte, aumentaban en 3,8\% las horas destinadas a "Seminario", porque este nuevo modelo planteaba su desarrollo a lo largo de todo el año académico. Las "Prácticas Profesionales" quedaban fuera de la contabilidad, pero se exigía su aprobación para la obtención del Título Profesional; se proponian otras 750 horas, de las cuales 400 estaban destinadas a "Práctica en Edificación" (300 horas en obra gruesa y 100 en terminaciones) y las 350 restantes, para "Práctica en Diseño". Además, se sumaban otras cinco asignaturas de formación general durante el transcurso de la carrera (Figura 6).

Esta estructura del plan de estudios -que disponía de un trimestre exclusivo

26 | Una hora pedagógica equivale a 50 minutos de hora cronológica. 
27 | En 1998 el Gobierno de Chile firmó un convenio con el Banco Mundial (BIRF) para implementar un programa de mejoramiento de la calidad de la educación terciaria, denominado "Programa de Mejoramiento de la Calidad y Equidad de la Educación (MECESUP)”.

28 Por entonces, integraba la Aristotle University de Thessaloniki (Grecia) y ejercía como Presidente de la Asociación Europea de Facultades de Arquitectura.

29 El Sistema único de Créditos Académicos Transferibles (SCT) fue establecido en 2006 por los rectores del $\mathrm{CRUCH}$; el sistema homologa, en todas las instituciones, los créditos de los planes de estudios de las distintas titulaciones. El SCT-Chile es equivalente al "European Credit Transfer and Accumulation System (ECTC)", sistema relacionado con la carga académica máxima que debe realizar el estudiante al semestre. para el trabajo en taller- implicaba que las horas consignadas al área de diseño de la EA UBB superaban con creces las de otras instituciones educativas.

En 1991 también se aprobó la nueva estructura orgánica de la FARCODI que quedó constituida por los Departamentos de Diseño y Teoría de la Arquitectura, de Planificación y Diseño Urbano, de Ciencias de la Construcción y de Comunicación Visual y por las Escuelas de Arquitectura, de Construcción Civil y de Diseño Gráfico, en Chillán. Igualmente, hubo que redistribuir a los(as) académicos(as) de acuerdo a los nuevos departamentos, elegir los cargos directores(as) de Departamento y nombrar los directores de esas escuelas. Los resultados de estas designaciones alcanzaron a Ricardo Hempel Hotzapel como Decano, a Ernesto Vilches Zapata como Secretario de Facultad y a quien esto escribe, como Director de la EA. Asimismo, y tal como se mencionó antes, ese año fue preparada la postulación ante el RIBA, acreditación alcanzada el mismo 91, y que posicionó a la EA como la primera carrera de Arquitectura del país en obtenerla y que aún mantiene.

\section{Avances institucionales previos al plan de 2014}

A partir de la acreditación internacional, la carrera de Arquitectura decidió someterse voluntariamente a distintos procedimientos evaluativos que aseguraban su calidad institucional educativa. Por un lado, postuló al proceso de acreditación nacional en 2003 (CNAP), siendo acreditada por 5 años inicialmente y reiterando este logro en tres oportunidades, además de incrementarlo a 6 años en la última ocasión (hasta el año 2021).

En 2004, la Facultad se adjudicó el proyecto "Construcción de una red de escuelas de arquitectura a nivel nacional con el propósito de innovar y desarrollar sus modelos curriculares a la luz de los acuerdos académicos internacionales, y de favorecer la interrelación docente y estudiantil” MECESUP ${ }^{27}$ UBB0404. La propuesta fue diseñada y presentada por el Decano de entonces, Ricardo Hempel Holzapfel, administrada en sus inicios por quien esto escribe y, en la fase final, continuada por Roberto Goycoolea Infante. Este fue el primer proyecto en red entre escuelas de Arquitectura nacionales y que asociaría a las de la Universidad de Valparaíso, la Pontificia Universidad Católica de Chile, la Universidad Arturo Prat y la UBB (encargada además de su gestión y administración). Las tareas concentraban discusiones y análisis en torno de las competencias profesionales del arquitecto, con la finalidad de diseñar un plan de estudio básico, denominado Plan de Estudio General (PEG) que compartirian las mallas curriculares de todas las carreras de Arquitectura del país.

Durante este periodo se participó en dos proyectos Alfa Tuning, el primero referente al levantamiento de las competencias generales $\circ$ transversales necesarias de considerar para la formación de los profesionales y, el segundo, focalizado en doce áreas formativas, entre las cuales estaba el área de Arquitectura, cuya finalidad fue la de alcanzar una convergencia en la definición de las competencias profesionales relativas a cada una de las titulaciones de esas áreas. Estos proyectos europeos tuvieron su versión Alfa Tuning América Latina fomentado por el MINEDUC y, por la convergencia con el MECESUP UBB0401, se solicitó a la UBB la designación de un representante nacional para el área de Arquitectura con el objetivo de consensuar, entre los países latinoamericanos partícipes, una definición de las competencias específicas y genéricas asociadas al perfil de egreso de arquitecto.

A raíz de ello la UBB convocaría, el 18 de enero de 2006, en la ciudad de Santiago, a todas las carreras de Arquitectura (públicas y privadas) del país a la "Jornada Temática Tuning América Latina". La finalidad fue acordar las competencias genéricas disciplinares del área, partiendo de una propuesta previa elaborada por el equipo del proyecto UBB0401. Los resultados fueron presentaron en la primera reunión del proyecto Alfa Tuning, efectuada en San José de Costa Rica, y publicados en el libro Reflexiones y Perspectivas de la Educación Superior en América Latina informe final-Proyecto Tuning-América Latina 2004-2007 (2007), texto más tarde complementado con los resúmenes de un segundo proyecto focalizado 
en la definición del meta-perfil del Arquitecto en América Latina: Educación Superior en América Latina: Reflexiones y Perspectivas en Arquitectura (Vélez, 2013).

Así, con el fin de implementar una formación basada en competencias en el currículo formativo del profesional arquitecto, se mantuvo por varios años una labor conjunta con representantes de facultades europeas y latinoamericanas. La experiencia Tuning y el contacto con el arquitecto Constantin Spiridonidis ${ }^{28}$ facilitaron la adjudicación de otros dos proyectos financiados por la Comunidad Europea: el ENHSA Latin America Proyecto Erasmus "Competences Based Architectural Curricula as tools for Quality and Mobility (2008-2011)" y el "ARCHI MUNDUS: Building up Quality in Architectural Education (2011-2013)", con destacada participación de académicos de la EA-UBB.

\section{Plan de estudios de 2014}

Después que la UBB obtuviera la primera acreditación institucional CNA en 2004, durante la rectoría del Hilario Hernández Gurruchaga, la institución determinó reformular su Modelo Educativo y orientarlo a una formación basada en competencias y resultados de aprendizajes, dando cuenta con ello de la política del MINEDUC en el ámbito de la Educación Superior. Con ese horizonte, en 2005, se conformó una comisión con académicos representantes de todas las facultades y cuyo objetivo fue definir las directrices para el diseño del nuevo Modelo Educativo institucional y, por lo tanto, afrontar la renovación curricular de todos sus programas de pregrado. Bajo la rectoría del arquitecto Héctor Gaete Feres, en 2008, fue establecido el nuevo Modelo Educativo Institucional, iniciando así el proceso de armonización curricular de todos los programas de pregrado.

Los nuevos planes de estudios debieron formular un perfil de egreso, definir las competencias especificas y genéricas asociadas a dicho perfil, incorporar los SCT-Chile ${ }^{29}$ y evaluar la posibilidad de ofrecer a los estudiantes salidas intermedias. Conjuntamente, debieron estructurarse en ciclos formativos, incorporar la articulación con el postgrado a nivel de magíster en las carreras que conducen al grado de licenciado, diseñar los programas de asignaturas considerando resultados de aprendizajes y elaborar guías didácticas que permitieran la bajada al aula de los respectivos programas.

Hasta su aprobación por la H. Junta Directiva, el 13 de enero del 2014, el proceso de rediseño del plan de estudios fue extenso e involucró a sucesivos directores de Escuela: los arquitectos Roberto Burdiles Allende y Hernán Barría Chateau. El plan fue consensuado con la comunidad académica de la EA y conformado a partir de la definición del perfil de egreso y de las competencias específicas y genéricas asociadas a dicho perfil. Su implementación se inició con los ingresantes de marzo de ese mismo año.

El Plan de estudios de 2014 se estructuró en base a dos ciclos: el Formativo Inicial con una duración de 3 años ( 6 semestres), coincidentes con el Plan de Estudio General (PEG) y elaborado a partir del MECESUP UBB0401; y el de Habilitación Profesional y Titulación, para el cuarto y el quinto año de la carrera, conducente a el Grado de Licenciado(a) en Ciencias Básicas de la Arquitectura y a la Titulación, respectivamente. Asimismo, el plan incorporó los SCT-Chile (30 SCT/ semestre y 300 SCT para toda la trayectoria formativa del Plan de Estudios) y consideró una salida intermedia -al cumplirse el quinto semestre y con 150 SCT aprobados- como Técnico(a) de Nivel Superior en Arquitectura.

En esta línea, el plan incluyó cuatro niveles de inglés comunicacional y asignaturas de formación integral, integró las prácticas profesionales dentro de la malla, estableció los perfiles de egreso del Licenciado(a) y del Técnico(a) de Nivel Superior en Arquitectura, articuló con el postgrado -a nivel de magíster- a través de asignaturas electivas de quinto año, incorporó asignaturas vinculadas al diseño bioclimático y, finalmente, redujo su extensión total a 5 años.

El Plan de 2014 priorizó temáticas contemporáneas y globales, emanadas de los problemas en Educación Superior y en formación profesional que trascendian la especificidad de cada país. Proyectos como el Tuning, ENHSA o 


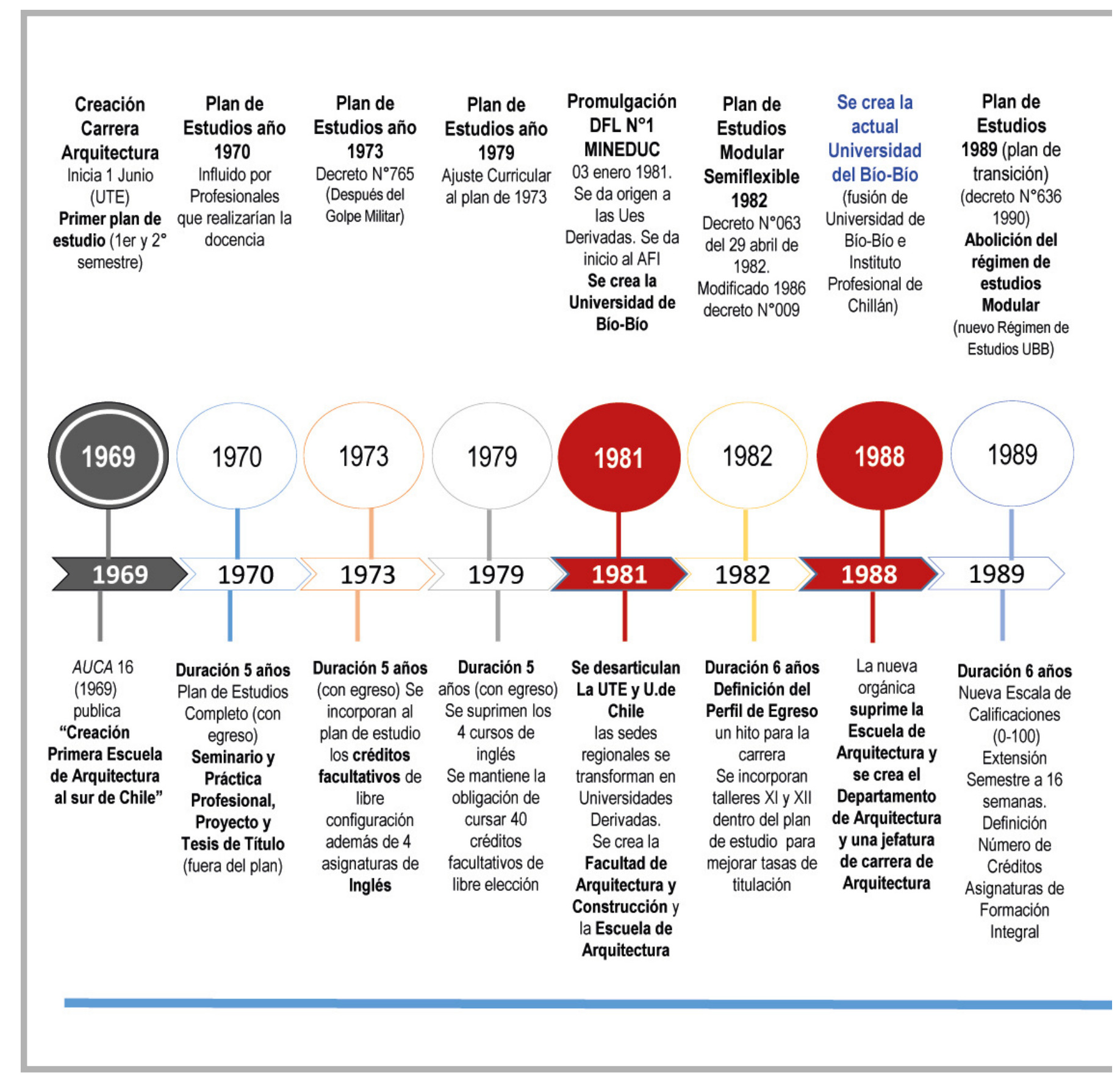

Figura 7.

50 años de la carrera de Arquitectura, Universidad del Bio-Bio: Planes de Estudios y estructuras curriculares. Fuente: Elaboración del autor 


$\begin{array}{ccc}\text { Organización y } & \text { Plan de Estudios } & \text { Acreditación } \\ \text { Estructura del } & 1991 & \text { nacional } \\ \text { Departamento } & \text { Decreto } N^{\circ} 1155 & \text { CNAP (2003) } \\ \text { de Arquitectura } & \text { (proceso ampliamente } & \text { Adjudicación } \\ \text { y Facultad de } & \text { participativo) } & \text { Mecesup UBB } \\ \text { Arquitectura } & \text { Plan Anual con } & 0404 \text { (2004) } \\ \text { Construcción y } & \text { organización } & \text { 1er Proyecto } \\ \text { Diseño } & \text { trimestral } & \text { Tuning- } \\ \text { Jornadas de } & \text { Se incorporan } & \text { Tún } \\ \text { revisión crítica } & \text { electivos } & \text { América Latina } \\ \text { del plan de } & \text { profesionales } & \text { (2004-2007) } \\ \text { estudio } & & \end{array}$

\begin{tabular}{|c|c|c|c|}
\hline $\begin{array}{l}\text { Se decreta el } \\
\text { Modelo } \\
\text { Educativo de } \\
\text { la UBB } \\
\text { Proyecto } \\
\text { ENHSA Latin } \\
\text { America } \\
\text { Erasmus } \\
\text { (2008-2011) }\end{array}$ & $\begin{array}{l}2^{\circ} \text { Proyecto } \\
\text { Tuning- } \\
\text { América Latina } \\
\text { (2011-2013) } \\
\text { Proyecto } \\
\text { ARCHI } \\
\text { MUNDUS } \\
\text { (2001-2013) }\end{array}$ & $\begin{array}{c}\text { Se Implementa } \\
\text { nuevo Plan de } \\
\text { Estudios } 2014 \\
\text { Ingreso de } \\
\text { primera cohorte } \\
\text { de estudiantes a } \\
\text { la nueva malla } \\
\text { curricular }\end{array}$ & $\begin{array}{c}50 \text { años de } \\
\text { la Carrera } \\
\text { de } \\
\text { Arquitectura } \\
(1969-2019)\end{array}$ \\
\hline
\end{tabular}

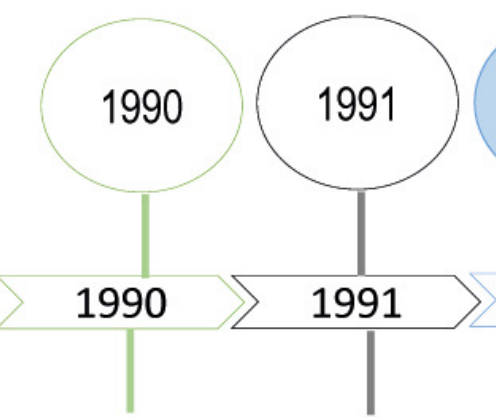

$\begin{array}{cc}\text { Elección Primer } & \text { Primera } \\ \text { Rector en } & \text { Acreditación } \\ \text { Democracia } & \text { Internacional de } \\ \text { (Agosto 1990) } & \text { la carrera de } \\ \text { 1990-1994 } & \text { Arquitectura } \\ & \text { (RIBA 1991) re- } \\ & \text { acreditación } \\ & \text { continua hasta } \\ & \text { el año 2020 }\end{array}$

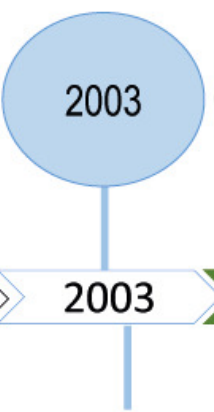

1 era.

Acreditación Nacional por la CNAP por 5 años (2003) re-acreditada hasta el 2021

Formulación de competencias especificas en trabajo en red con 4 escuelas (PUC, U.V, UAP, UBB)

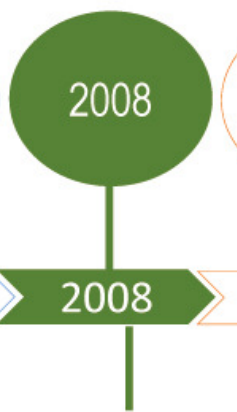

Se inicia el proceso de Renovación curricular en la UBB

Se comienza la formulación de un nuevo plan de estudios. Se incorporan los SCT- CHILE

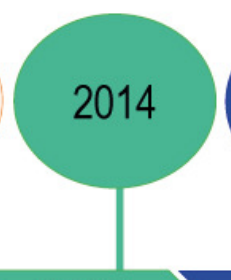

\section{1}

2014

2019

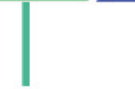

Definición de Plan de estudio Ranking meta-perfil del con SCT América Economía. Arquitecto

Salida Sitúa a la carrera intermedia de Arquitectura de la UBB en $3^{\circ}$ lugar a nivel de pais,entre 30 escuelas de arquitectura. Primeros egresados plan de estudio 2014 
ACHIMUNDUS facilitaron plataformas de discusión conjunta sobre la formación del arquitecto, lo hicieron con una perspectiva más amplia y propia de una sociedad basada en el conocimiento y en la que, el campo profesional de la arquitectura, se diversifica y amplía con nichos emergentes.

Otro factor que influyó en este nuevo plan de estudios fue que muchos académicos de la Facultad desarrollaron programas de postgrado, magíster o doctorado en el extranjero y regresaron con experiencias que se plasmaron en esta nueva oferta formativa; y ciertos aspectos muy fuertes en los planes iniciales giraron hacia una formación reflexiva, donde la investigación surgió como una opción laboral importante para los egresados. Ya la formación de pregrado no era suficiente, las opciones de magíster y de doctorado resultaban un camino concreto y anhelado por los titulados para complementar su formación profesional.

El nuevo Plan de estudios fortaleció la línea de "Representación Arquitectónica", pero suprimió la formación inicial en plástica tradicional (Composición Arquitectónica y Configuración Espacial), incorporó "Diseño Bioclimático" bajo el concepto de una arquitectura sustentable y sostenible, fortaleció al estudiante en la teoria y la reflexión a través de una secuencia de asignaturas denominada "Fundamentos de Arquitectura" que culminaba con "Seminario" como requisito para la obtención del grado de licenciado.

$\mathrm{Si}$ se analiza el peso de las distintas áreas formativas entre el nuevo plan y el de 1991, el primero decrecía un 13,4\% en el área de diseño (solo con 52\%), en el área técnica (estructura edificación, especialidades) mantuvo similares valores (en torno al 16\% del total), en la de historia y teoría se incrementó en un 2,9\%, debido a la incorporación de la línea de "Fundamentos de la Arquitectura". Disminuyeron, por su parte, urbanismo, en un 1,2\%, metodología de la investigación y seminario, en un 1,6\%; pero se incrementaron "Formación Integral", en 10\%, los electivos profesionales, en 4,8\% -gracias a la articulación con posgrado-, y "Práctica profesional" alcanzaba ahora una incidencia de un 3,3\%.

El Sistema de Créditos Transferibles SCT-Chile exige que todas las actividades curriculares que desarrolla el estudiante en su trayectoria formativa estén consideradas dentro del plan de estudios de la carrera, ello sin exceder los 30 SCT semestrales y 60 anuales, por ende, las prácticas profesionales, las asignaturas de formación integral que en los planes de estudios anteriores quedaban como exigencias a cumplirse y no formaban parte del currículo de la carrera, ahora debieron considerarse dentro del plan. A lo anterior se sumaba la reducción de la extensión formal del plan de estudios de la carrera de 6 a 5 años, lo que justifica la disminución porcentual del área de diseño, en comparación con el Plan de 1991.

Al momento de la edición de esta revista, se titularán los primeros arquitectos ajustados al Plan de estudios de 2014, lo que brindará la oportunidad de evaluar las innovaciones que se incorporaron y de verificar si tuvieron el impacto formativo deseado. Un plan de estudio, tal como se expresó al inicio de este texto, que ha resultado de la construcción de la trayectoria formativa, necesaria para lograr en el estudiante las competencias declaradas en el perfil de egreso. El proceso de evaluación implicará, ciertamente, disponer de mecanismos que permitan evidenciar si estas competencias se han adquirido y en qué medida lo han hecho al momento de recibir el título que habilita para ejercer la profesión. Es de responsabilidad institucional garantizar que los profesionales egresados cumplan con lo que la misma universidad ha declarado formar (Figura 7).

\section{Reflexiones finales}

Los cincuenta años de existencia de la carrera de Arquitectura de la UBB la encuentran en una categoria relevante a nivel nacional. Efectivamente, durante 2018 , la revista América Economía la situó tercera entre las mejores escuelas del país, corroborando con ello que el esfuerzo realizado por académicos, docentes y profesores, desde el primer Plan de estudios de 1969 al último de २014, ha sido exitoso y reconocido además por organismos e instituciones externas de aseguramiento de la calidad, como la CNA y el RIBA.

Esta retrospectiva de la carrera de Arquitectura a través de sus distintos planes de estudios permite advertir ciertas invariantes presentes desde sus 
inicios. Entre ellas, el carácter regional, vigente desde la fundación de la Escuela y que AUCA anticipara, que se mantiene presente en el perfil de egreso, como también el entendimiento de que la arquitectura es obra construible y habitable, a lo cual se le ha añadido hoy el concepto de "sustentable".

Cabe mencionar que ocho planes de estudios en 50 años de trayectoria formativa, promedia un ajuste curricular aproximadamente cada seis años $y$, si se considera que la duración formal de la carrera está en torno a los 6 años, parecen pertinentes los lapsos de revisiones y/o reformulaciones de los planes formativos, de manera que se vayan adecuando a variables contextuales y dinámicas de la disciplina.

Asimismo, la retroalimentación continua de los perfiles de egreso y las trayectorias formativas expresadas en los planes de estudios de la carrera han resultado claves en el éxito de la carrera de Arquitectura de la UBB en el concierto nacional.

Por último, huelga expresar que sin un equipo de académicos y docentes comprometidos con este proyecto común que es la formación de arquitectos en la UBB, sin el liderazgo claro y potente que ejercieran los distintos directores y directoras de la Escuela durante estos cincuenta años, la carrera de Arquitectura y la Escuela que la administra no habrian alcanzado el posicionamiento y el prestigio que ostenta al día de hoy.

\section{Referencias}

(1982). Definición de las metas, objetivos y perfil de los Módulos Básico, Medio y Avanzado del programa de Arquitectura. Concepción: Dirección de Escuela de Arquitectura, Universidad del Bío-Bío.

(1989). Segundas Jornadas de estudio crítico en torno a la enseñanza de la Arquitectura. Concepción: Dirección de Escuela de Arquitectura, Universidad del Bío-Bío.

Beneitone, P., Esquetini, C., González, J., Marty Maletá, M., Siufi, G., Wagenaar, R. (2007). Reflexiones y Perspectivas de la Educación Superior en América Latina Informe final- Proyecto Tuning- América Latina 2004-2007. Bilbao: Universidad de Deusto.

Aptecar González, B. (1984). Breve reseña sobre la Escuela de Arquitectura. Concepción: Dirección de Escuela de Arquitectura, Universidad del Bío-Bío.

Comisión Nacional de Acreditación (2015). Glosario de términos complementarios criterios de acreditación de pregrado. Recuperado de https://www.cnachile. cl/Documentos\%20de\%20Paginas/Glosario\%20Pregrado.pdf

Decreto No 1221. Santiago, 10 de julio de 1969.

Goycoolea Infante, R. (2017). Escuela de Arquitectura. UBB desde la memoria. Concepción: Ediciones Universidad del Bío-Bío.

Goycoolea Infante, R. y Lobos Lápera, V. (1976). Reglamento de Créditos Facultativos. Concepción: UTE.

Lobos Lápera, V. (s/f). Memoria Explicativa del Plan de estudios de Arquitectura. Concepción: Documento interno de la Escuela de Arquitectura, Universidad Técnica del Estado.

Meissner, E. (1984). La configuración espacial sobre estructuras configuradoras y espacios configurados. Concepción: Universidad del Bío-Bío.

Maissner, E. (2003). Teoría del signo en Arquitectura (Charlas de Nottingham). Concepción: Universidad de Concepción.

Muñoz, M. D. (c. 1985). Premios Nacionales de Arquitectura, 1969-1985. Concepción: Facultad de Arquitectura y Construcción, Universidad del Bío-Bío.

Shapira, A. (Ed.) (1969). Nueva escuela de arquitectura: Concepción, AUCA (16), 4. Valassina Simonetta, F. (1987). Definición de los Módulos Básico, Medio y Avanzado y la Licenciatura del programa de Arquitectura ( 18 de noviembre). Concepción: Facultad de Arquitectura y Construcción, Universidad del Bío-Bío.

Vélez González, S. (coord.) (2013). Educación Superior en América Latina: Reflexiones y Perspectivas en Arquitectura (2013). Bilbao: Universidad de Deusto. 